\title{
Heavy quark energy loss far from equilibrium in a strongly coupled collision
}

\author{
Paul M. Chesler, ${ }^{a}$ Mindaugas Lekaveckas ${ }^{a, b}$ and Krishna Rajagopal ${ }^{a, b}$ \\ ${ }^{a}$ Center for Theoretical Physics, Massachusetts Institute of Technology, \\ Cambridge, MA 02139, U.S.A. \\ ${ }^{b}$ Physics Department, Theory Unit, CERN, \\ CH-1211 Genève 23, Switzerland \\ E-mail: pchesler@mit.edu, lekaveck@mit.edu, krishna@mit.edu
}

ABSTRACT: We compute and study the drag force acting on a heavy quark propagating through the matter produced in the collision of two sheets of energy in a strongly coupled gauge theory that can be analyzed holographically. Although this matter is initially far from equilibrium, we find that the equilibrium expression for heavy quark energy loss in a homogeneous strongly coupled plasma with the same instantaneous energy density or pressure as that at the location of the quark describes many qualitative features of our results. One interesting exception is that there is a time delay after the initial collision before the heavy quark energy loss becomes significant. At later times, once a liquid plasma described by viscous hydrodynamics has formed, expressions based upon assuming instantaneous homogeneity and equilibrium provide a semi-quantitative description of our results - as long as the rapidity of the heavy quark is not too large. For a heavy quark with large rapidity, the gradients in the velocity of the hydrodynamic fluid result in qualitative consequences for the 'drag' force acting on the quark. In certain circumstances, the force required to drag the quark through the plasma can point opposite to the velocity of the quark, meaning that the force that the plasma exerts on a quark moving through it acts in the same direction as its velocity. And, generically, the force includes a component perpendicular to the direction of motion of the quark. Our results support a straightforward approach to modeling the drag on, and energy loss of, heavy quarks with modest rapidity in heavy ion collisions, both before and after the quark-gluon plasma hydrodynamizes, and provide cautionary lessons at higher rapidity.

KEYwords: Holography and quark-gluon plasmas, Quark-Gluon Plasma, Gauge-gravity correspondence

ARXIV EPRINT: 1306.0564 


\section{Contents}

1 Introduction 1

2 Holographic description $\quad 4$

2.1 Gravitational description of colliding sheets of energy 4

2.2 String dynamics 9

2.3 Extracting the drag force acting on the quark from the string profile 12

3 Results $\quad 14$

$\begin{array}{lll}3.1 & \text { Heavy quark with zero rapidity } & 14\end{array}$

3.2 Heavy quark with zero transverse momentum 20

3.3 Heavy quark with nonzero rapidity and transverse momentum 26

4 Conclusions and lessons for heavy ion physics 31

$\begin{array}{ll}\text { A Trailing string solution in an equilibrium background } & 33\end{array}$

$\begin{array}{ll}\text { B Varying temperature } & 34\end{array}$

\section{Introduction}

The discovery that strongly coupled quark-gluon plasma (QGP) is produced in ultrarelativistic heavy ion collisions has prompted much interest in the real-time dynamics of strongly coupled non-Abelian plasmas. For example, heavy quark energy loss has received substantial attention. If one shoots a heavy quark through a non-Abelian plasma, how much energy does it lose as it propagates? Equivalently, what is the drag force required to pull a heavy quark through such a plasma at a specified velocity? This question has been answered [1-3] for homogeneous plasma in thermal equilibrium in strongly coupled $\mathcal{N}=4$ supersymmetric Yang-Mills (SYM) theory in the large number of colors $\left(N_{c}\right)$ limit, where holography permits a semiclassical description of energy loss in terms of string dynamics in asymptotically $\mathrm{AdS}_{5}$ spacetime [1-6]. One challenge (not the only one) in using these results to glean qualitative insights into heavy quark energy loss in heavy ion collisions is that a heavy quark produced during the initial collision event must first propagate through the initially far-from-equilibrium matter produced in the collision before it later plows through the expanding, cooling, hydrodynamic fluid of strongly coupled QGP. In this paper we shall describe calculations that provide some qualitative guidance for how to meet this challenge. For a review of many other ways in which holographic calculations have yielded qualitative insights into properties of strongly coupled QGP and dynamics in heavy ion collisions, see ref. [7]. 
We want a toy model in which we can reliably calculate how the energy loss rate of a heavy quark moving through the far-from-equilibrium matter present just after a collision compares to that in strongly coupled plasma close to equilibrium. We study the energy loss of a heavy quark moving through the debris produced by the collision of planar sheets of energy in strongly coupled SYM theory introduced in ref. [8] and analyzed there and in refs. $[9,10]$. The incident sheets of energy move at the speed of light in the $+z$ and $-z$ directions and collide at $z=0$ at time $t=0$. They each have a Gaussian profile in the $z$ direction and are translationally invariant in the two directions $\vec{x}_{\perp}=x, y$ orthogonal to $z$. Their energy density per unit transverse area is $\mu^{3}\left(N_{c}^{2} / 2 \pi^{2}\right)$, with $\mu$ an arbitrary scale with respect to which all dimensionful quantities in the conformal theory that we are working in can be measured. The width $w$ of the Gaussian energy-density profile of each sheet is chosen to be $w=1 /(2 \mu)$. We shall describe this setup, and its holographic description, in section 2.1. Although there is no single right way to compare the widths of these translationally invariant sheets of energy with Gaussian profiles to the widths of a nucleus that has been Lorentz-contracted by a factor of 107 (RHIC) or 1470 (LHC), reasonable estimates suggest that our choice of $w \mu$ corresponds to sheets with a thickness somewhere between the thickness of the incident nuclei at RHIC and the LHC [8]. The recent investigations of refs. $[9,10]$ suggest that it would be interesting to repeat our analyses for varying values of $w \mu$, but we leave this for future work since here we shall only be seeking to draw qualitative lessons.

The principal lesson that has been learned to date from analyses of the collisions of strongly coupled sheets of energy as in refs. [8-10] and from many other analyses of how strongly coupled plasma forms from a large number of widely varied far-from-equilibrium strongly coupled initial collisions (for example, see refs. [11-18]) is that the fluid hydrodynamizes, i.e. comes to be described well by viscous hydrodynamics, after a time $t_{\text {hydro }}$ that is at most around $(0.7-1) / T_{\text {hydro }}$, where $T_{\text {hydro }}$ is the effective temperature (for example, defined from the fourth root of the energy density) at the hydrodynamization time $t_{\text {hydro. }}$ At $t_{\text {hydro }}$, the fluid can still have sufficiently large velocity gradients and pressure anisotropies that the dissipative effects of viscosity are significant. In the context of hydrodynamic modeling of heavy ion collisions at RHIC (for a recent example see ref. [19]) $t_{\text {hydro }} \sim 0.7 / T_{\text {hydro }}$ corresponds to a time $\sim 0.3 \mathrm{fm} / c$ when $T \sim 500 \mathrm{MeV}$. This is about a factor of two earlier in time than the upper bounds on the hydrodynamization times inferred from hydrodynamic modeling of RHIC collisions [19-21]. Because QCD is asymptotically free, the dominant dynamics at the earliest moments of a sufficiently energetic heavy ion collision are expected to be weakly coupled, with the relevant (weak) coupling being $\alpha_{\mathrm{QCD}}$ evaluated at the (short) distance scale corresponding to the mean spacing between gluons in the transverse plane at the moment when the two highly Lorentz-contracted nuclei collide. So, it would be inappropriate to take the estimates obtained in a context in which the colliding sheets of matter are strongly coupled from beginning to end as estimates for the hydrodynamization times of heavy ion collisions per se. The impact of these estimates is that they teach us that the $\sim 10$ year old result $[20,21]$ that the matter produced in RHIC collisions takes at most $0.6-1 \mathrm{fm} / c$ to hydrodynamize should not be seen as 'rapid thermalization' since this timescale is comfortably longer than what we now know to expect 
if the physics of heavy ion collisions were strongly coupled from the start. After we have calculated the drag force on a heavy quark that finds itself in the midst of the colliding sheets of energy density, we shall seek similarly qualitative lessons to those that have been drawn from the analyses of the collisions themselves.

We compute heavy quark energy loss by inserting a heavy quark moving at constant velocity $\vec{\beta}$ between the colliding sheets before the collision and calculating the force needed to keep its velocity constant throughout the collision. Via holography, the colliding planar sheets of energy in SYM theory map into colliding planar gravitational waves in asymptotically $\mathrm{AdS}_{5}$ spacetime [8]. The addition of a heavy quark moving at constant velocity $\vec{\beta}$ amounts to including a classical string attached to the boundary of the geometry [6] and dragging the string endpoint at constant velocity $\vec{\beta}$, pulling the string through the colliding gravitational wave geometry. We show how to compute the profile of the string in this dynamical background in section 2.2. The force needed to maintain the velocity of the string endpoint, which we compute in section 2.3 , yields the energy loss rate of the heavy quark $[1,2]$.

We describe our results in section III, beginning in section 3.1 with the case in which the heavy quark is moving with $\vec{\beta}$ perpendicular to the $z$-direction, meaning that it has zero rapidity. We compare the drag force that we calculate to what it would be in a homogeneous plasma in thermal equilibrium that has the same energy density or transverse pressure or longitudinal pressure as the matter that the quark finds itself in at a given instant in time. We find that the peak value of the drag force, which occurs at a time when the matter produced in the collision is still far from equilibrium, is comparable to the peak value of the drag force in a static plasma with the same instantaneous energy density or pressure. However, we find that both the initial rise in the drag force and its peak are delayed in time relative to what they would be in a static plasma with the same instantaneous energy density or pressure. In appendix B we provide some evidence that this time delay is of order $1 / \pi T_{\text {hydro }}$ at low $\beta$ and increases slowly as $\gamma \equiv 1 / \sqrt{1-\beta^{2}}$ increases. All these results are robust, in particular in the sense that we see them again when we consider a heavy quark moving with some nonzero rapidity in sections 3.2 and 3.3.

The message from our results at early times is that there is no sign of any enhancement in the energy loss experienced by a heavy quark by virtue of the matter that it finds itself moving through being far from equilibrium. In broad terms, the energy loss is comparable to what it would be in an equilibrium plasma with the same energy density; when looked at in more detail, it can be significantly less by virtue of the initial delay in its rise. This is quite different than at weak coupling, where instabilities in the far-from-equilibrium matter can arise and can result in substantially enhanced rates of heavy-quark energy loss [22]. There are, however, no signs of any instabilities in the debris produced in the collisions of the sheets of strongly coupled matter that we analyze $[8,9]$ or, for that matter, in any analyses of far-from-equilibrium strongly coupled matter to date.

When we look at the drag force at late times, after the strongly coupled fluid has hydrodynamized, we find different results depending on whether the heavy quark has small or large rapidity. At small rapidity, the drag force that we calculate is described semiquantitatively by assuming a homogeneous plasma in equilibrium with an appropriate time- 
dependent temperature. At large rapidity, however, this approximation misses qualitative effects that, we show in sections 3.2 and 3.3, can be attributed to the presence of gradients in the fluid velocity. We find that a velocity gradient in the fluid has the greatest effect on the energy loss of the heavy quark when the direction of motion of the heavy quark is most closely aligned with the velocity gradient. As a consequence, effects of velocity gradients are larger at larger rapidity. We find generically that the force that must be exerted on the quark in order to move it along its trajectory includes a component perpendicular to the direction of motion of the quark, a component that can be substantial in magnitude. In certain cases we also find that, as a consequence of gradients in the fluid velocity, the $z$-component of the force that is required to move the quark in the positive- $z$ direction points toward negative $z$ ! We conclude in section IV with a look at the lessons on how best to model the drag on heavy quarks produced in heavy ion collisions that can be drawn from our results.

\section{Holographic description}

In a strongly coupled conformal gauge theory with a dual gravitational description, a heavy quark moving through out-of-equilibrium matter that is on its way to becoming strongly coupled plasma consists of a string moving in some non-equilibrium, but asymptotically $\mathrm{AdS}_{5}$, black brane spacetime. For an infinitely massive quark, the endpoint of the string is attached to the four-dimensional boundary of the geometry. The geometry of the boundary is that of Minkowski space and the trajectory of the string endpoint on the boundary coincides with the trajectory of the quark.

We shall focus on the case in which the gauge theory is $\mathcal{N}=4 \mathrm{SYM}$ theory with $N_{c}$ colors, although our results can immediately be generalized to any conformal gauge theory with a gravity dual upon making a suitable modification to the relationship between the 't Hooft coupling $\lambda \equiv g^{2} N_{c}$ of the gauge theory with $g$ the gauge coupling constant, and corresponding quantities in the gravity dual. In the limit of large $N_{c}$ and large $\lambda$, the evolution of the black brane geometry is governed by Einstein's equations

$$
R_{M N}-\frac{1}{2} G_{M N}(R-2 \Lambda)=0,
$$

with cosmological constant $\Lambda=-6$. In this limit, the back reaction of the string on the geometry is negligible meaning that we can solve Einstein's equations first, independently of the string equations, and then subsequently determine the shape of the string in a background given by the solution to Einstein's equations.

\subsection{Gravitational description of colliding sheets of energy}

The geometry we choose to study is that of the colliding gravitational shockwaves studied in ref. [8]. In the dual gauge theory living on the boundary this geometry corresponds to colliding planar sheets of energy density. In Fefferman-Graham coordinates the pre-collision metric is given by [23]

$$
d s^{2}=\frac{-d x_{+} d x_{-}+d \vec{x}_{\perp}^{2}+u^{4}\left[h_{+} d x_{+}^{2}+h_{-} d x_{-}^{2}\right]+d u^{2}}{u^{2}}
$$




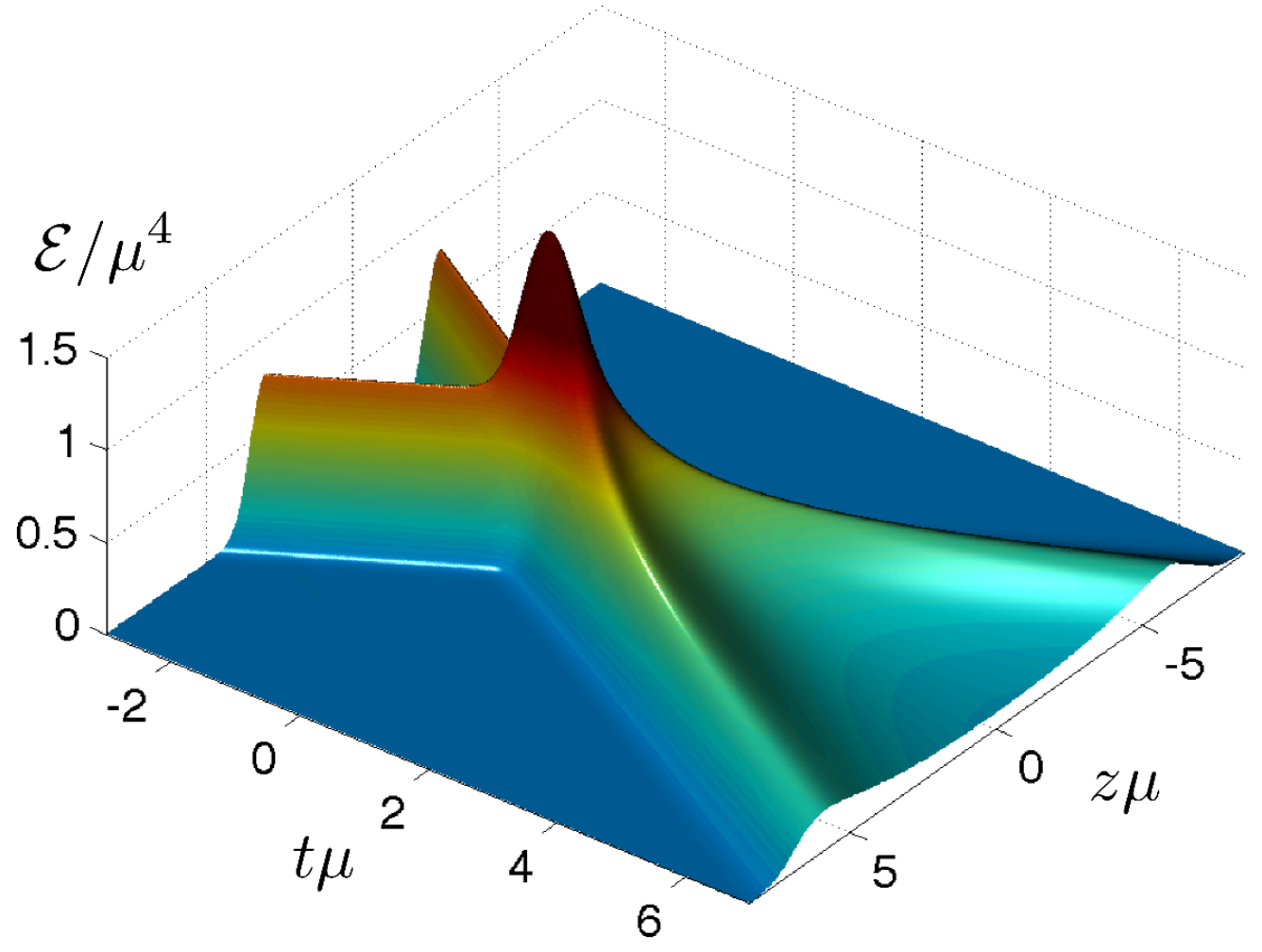

Figure 1. A plot of the boundary energy density as a function of time $t$ and position along the 'beam' direction $z$ for the colliding sheets of energy. Before $t=0$, the energy density is that of two sheets with Gaussian profiles moving toward each other at the speed of light. The sheets collide around time $t=0$ and leave debris in the forward light-cone that subsequently hydrodynamizes as it expands, becoming strongly coupled, liquid, plasma.

where $x_{ \pm}=t \pm z$ and $h_{ \pm} \equiv h\left(x_{ \pm}\right)$for some function $h\left(x_{ \pm}\right)$that specifies the profile of the incident gravitational shockwaves and hence the incident sheets of energy in the boundary theory. The boundary of the geometry is located at AdS radial coordinate $u=0$. The gravitational shockwaves move in the $\pm z$ direction at the speed of light. If $h\left(x_{ \pm}\right)$has compact support then $h\left(x_{+}\right)$and $h\left(x_{-}\right)$do not overlap in the distant past and the metric (2.2) is an exact pre-collision solution to Einstein's equations. Following ref. [8], we choose Gaussian profiles

$$
h\left(x_{ \pm}\right)=\mu^{3}\left(2 \pi w^{2}\right)^{-1 / 2} e^{-\frac{1}{2} x_{ \pm}^{2} / w^{2}},
$$

where $\mu$ defines the energy scale, meaning that we shall measure all other dimensionful quantities in units of $\mu$.

For a given solution to Einstein's equations, the near-boundary behavior of the metric encodes the boundary stress tensor $T^{\mu \nu}[24,25]$. In the boundary gauge theory the energy density of each shock is proportional $h\left(x_{ \pm}\right)$[23]. The metric that we have described 
therefore corresponds in the boundary theory to two sheets of energy density (infinite in extent, and translation-invariant, in the transverse dimensions) that are moving towards each other in the $\pm z$ directions at the speed of light. The energy density profiles of the sheets are Gaussians with widths $w$, and the incident sheets each have an energy per unit area that is given by $N_{c}^{2} \mu^{3} /\left(2 \pi^{2}\right)$ [8]. We choose the width of each shock to be $w=0.5 / \mu$, meaning that we shall be probing the collision of sheets of energy that are thinner by a factor of $2 / 3$ than those in ref. [8].

Near the collision time, when the functions $h\left(x_{ \pm}\right)$begin to overlap, the metric $(2.2)$ ceases to be a solution to Einstein's equations. Using (2.2) as initial data in the distant past, one must therefore compute the future evolution of the geometry numerically. Our numerical scheme for solving Einstein's equations can be found in refs. [8, 10]. In what follows we simply state some of the salient features. A useful choice of coordinates for the numerical evolution is that of infalling Eddington-Finkelstein coordinates where the metric takes the form

$$
d s^{2}=\frac{-A d t^{2}+\Sigma^{2}\left[e^{B} d \vec{x}_{\perp}^{2}+e^{-2 B} d z^{2}\right]+2 d t(F d z-d u)}{u^{2}}
$$

where $A, B, \Sigma$ and $F$ are functions of $t, z$ and $u$. Lines of constant time $t$ (and spatial coordinates $\vec{x}_{\perp}$ and $z$ ) are infalling null geodesics. ${ }^{1}$

An important practical matter when solving Einstein's equations is fixing the computational domain in $u$. The geometry we study in this paper contains a black brane with planar topology. Moreover, the event horizon exists in the infinite past, even before the collision takes place on the boundary $[8,12]$. Therefore, a natural choice is to excise the geometry inside the horizon, as this region is causally disconnected from the outside geometry. To perform the excision we identify the location of an apparent horizon (which always lies inside the event horizon) and choose to stop integrating Einstein's equations any further into the black brane at its location. Our choice of coordinates makes this procedure particularly simple. The metric ansatz (2.4) is invariant under the residual diffeomorphism

$$
\frac{1}{u} \rightarrow \frac{1}{u}+\xi(t, z)
$$

where $\xi$ does not depend on the radial coordinate $u$ but is an arbitrary function of the boundary spacetime coordinates $t$ and $z$. One may fix $\xi$ by demanding that the location of the apparent horizon be at $u=1$. With this choice of coordinates, the boundaries of the computational domain are static: $0<u<1$. The numerical procedure for determining $\xi$ and solving Einstein's equations is described in refs. $[8,10]$ and we shall not review it here. Following this procedure yields $\xi$ as well as the bulk metric functions $A, B, \Sigma$ and $F$, whose asymptotic near-boundary behavior determines the stress-energy tensor $T^{\mu \nu}$ of the colliding sheets of energy in the boundary gauge theory, as described in ref. [8].

\footnotetext{
${ }^{1}$ To the best of our knowledge there does not exist a closed form coordinate transformation taking the pre-collision metric (2.2) onto the metric (2.4) used for numerical evolution. Therefore, for initial data we compute the coordinate transformation required to put the initial metric (2.2) in the form of (2.4) numerically. For details see refs. [8, 10].
} 
In the distant past the apparent horizon lies very deep in the bulk. This presents a computational problem for solving Einstein's equations numerically as the coefficient functions in the metric (2.2) diverge deep in the bulk. As described in ref. [8], to regulate this problem we choose to study study shocks which propagate and collide in a low temperature background plasma of temperature $T_{\text {background }}$. In the distant past the effect of the background temperature is to push the apparent horizon up towards the boundary and thereby control the size of the metric coefficient functions deep in the bulk. Our choice of background temperature is $T_{\text {background }}=0.085 \mu$ which corresponds to an initial background energy density 213 times smaller than the energy density at the center of the sheets of energy and 450 times smaller than the energy density at $t=z=0$, during the collision.

Figure 1 shows a plot of the rescaled energy density

$$
\mathcal{E} \equiv \frac{2 \pi^{2}}{N_{\mathrm{c}}^{2}} T^{00}
$$

for our colliding sheets as a function of position and time. Before $t=0$, the energy density is that of two sheets of energy with Gaussian profiles moving toward each other at the speed of light. The sheets collide around time $t=0$ and leave debris in the forward light-cone. We shall calculate the rate of energy loss of (i.e. the 'drag force' acting on) a heavy quark moving through the far-from-equilibrium matter right near $t=z=0$, during the collision when the energy density is largest. We shall then follow the quark forward in time and see how it loses energy in the expanding, cooling, fluid that forms in the forward lightcone. In gravitational terms, we wish to study the dynamics of a string moving in the black brane geometry during and after the collision.

After the collision, the fluid in the forward lightcone is expanding meaning that at $z \neq 0$ it has a velocity that points in the direction of increasing $|z|$. The fluid velocity is defined to be the future-directed time-like eigenvector of $T_{\nu}^{\mu}$, normalized such that $u_{\mu} u^{\mu}=-1$ :

$$
T_{\nu}^{\mu} u^{\nu}=-\frac{N_{c}^{2}}{2 \pi^{2}} \varepsilon u^{\mu},
$$

with $-\varepsilon$ the eigenvalue, $\varepsilon$ being the proper energy density rescaled as in (2.6). At any spacetime point, we can find the local fluid rest frame by first using (2.7) to determine $u_{\text {lab frame }}^{\mu}$ and then boosting to a frame in which, at this spacetime point, $u^{\mu}$ is given by $u_{\text {rest frame }}^{\mu}=(1,0,0,0)$. In this local fluid rest frame, the stress-energy tensor at the spacetime point of interest is diagonal and can be written as

$$
T_{\text {rest frame }}^{\mu \nu}=\frac{N_{c}^{2}}{2 \pi^{2}} \operatorname{diag}\left(\varepsilon, \mathcal{P}_{\perp}, \mathcal{P}_{\perp}, \mathcal{P}_{\|}\right) .
$$

Note that at $z=0$ the velocity of the fluid vanishes by symmetry, meaning that at $z=0$ in (2.7) we have simply $u_{\text {lab frame }}^{\mu}=u_{\text {rest frame }}^{\mu}=(1,0,0,0)$ and $\varepsilon=\mathcal{E}=T^{00}\left(2 \pi^{2}\right) / N_{c}^{2}$.

Before we turn to describing the dynamics of the string and then to calculating the rate at which the heavy quark loses energy, we should justify referring to the matter that is formed in the forward light-cone as a fluid. In figure 2 we compare the pressures in the 'beam' direction and perpendicular to it at $z=0$ to what those pressures would 


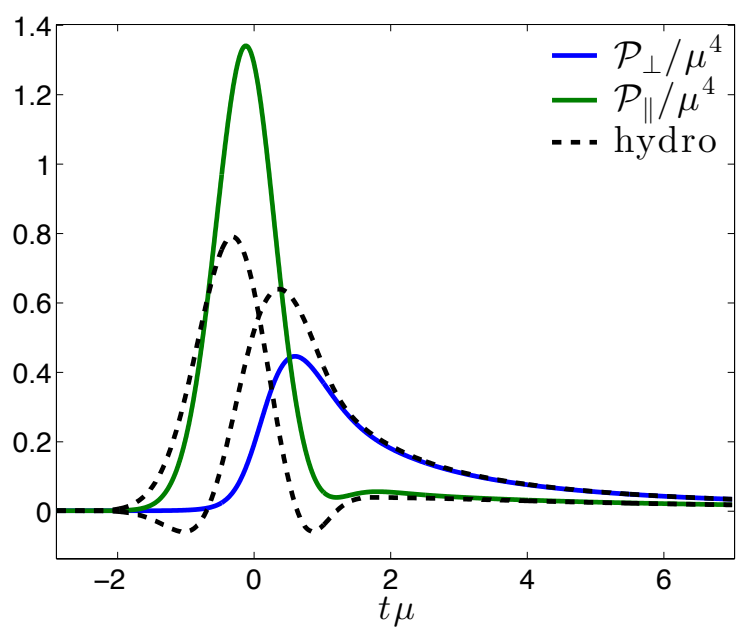

Figure 2. Pressure, both parallel to the direction of motion of the colliding sheets $\left(\mathcal{P}_{\|}\right)$and in the transverse directions $\left(\mathcal{P}_{\perp}\right)$, as a function of time at $z=0$ during the collision of the sheets of energy illustrated in figure 1. We compare the pressures in the collision, shown as solid colored curves, to what they would be if the hydrodynamic constitutive relations were satisfied, shown as dashed curves. We see that the strongly coupled fluid produced in the collision hydrodynamizes to a reasonable degree before $t=2 / \mu$, although with the more strict operational criterion defined in the text, the hydrodynamization time is $t_{\text {hydro }}=2.8 / \mu$.

be if the matter were a fluid described by the equations of viscous hydrodynamics for the $\mathcal{N}=4 \mathrm{SYM}$ plasma, to first order in a derivative expansion. In particular, the hydrodynamic constitutive relations allow us to determine $\mathcal{P}_{\|}$and $\mathcal{P}_{\perp}$ from the proper energy density $\varepsilon$ and the fluid velocity $u^{\mu}$, determined via (2.7). (Because we are making this comparison at $z=0$, where the fluid is at rest in the lab frame, in this instance (2.7) is trivial as described above.) We plot the pressures determined by applying the first order hydrodynamic constitutive relations to $\varepsilon$ and $u^{\mu}$ as dashed curves in figure 2 . We see that from a time that is clearly before $2 / \mu$ onwards the flow of the fluid is described very well by hydrodynamics. We can use the same operational definition of the hydrodynamization time $t_{\text {hydro }}$ as in ref. [8], namely the time after which the actual pressures within the matter produced in the initially far-from-equilibrium collision are both within $15 \%$ of the values derived from the hydrodynamic constitutive relations. With this definition, in figure 2 hydrodynamization occurs at $t=t_{\text {hydro }}=2.8 / \mu$. It is clear from the figure that one could choose a reasonable but less strict criterion for hydrodynamization with respect to which hydrodynamization occurs well before $2 / \mu$. The reason that with the strict criterion $t_{\text {hydro }}$ is a little later is that $\mathcal{P}_{\|}$is so small that a $15 \%$ relative deviation corresponds to a very small absolute deviation.

We shall calculate the drag force on the heavy quark as it plows through the matter produced in the collision both before the hydrodynamization time $t_{\text {hydro }}$, when the matter is far from equilibrium, and after $t_{\text {hydro }}$ when we have an expanding, cooling, hydrodynamic liquid. As in ref. [8], we see from figure 2 that at the hydrodynamization time $t_{\text {hydro }}$ the 
parallel and transverse pressures are very different. The fluid is very anisotropic at the time that it hydrodynamizes and becomes locally isotropic only at a much later time, beyond that shown in the figure. We shall therefore be studying the drag force on a heavy quark when it is first in the far-from-equilibrium matter at $t \sim 0$, well before hydrodynamization, and then later in an expanding cooling hydrodynamic fluid that is far from isotropic.

\section{$2.2 \quad$ String dynamics}

For a given solution to Einstein's equations, for example the one above that describes the dynamics of a black brane in the bulk and the collision, hydrodynamization, and hydrodynamic flow of the strongly coupled matter in the boundary theory, we can add an infinitely heavy quark moving through the boundary theory matter by adding a string in the bulk geometry whose endpoint follows the trajectory of the quark along the boundary. The dynamics of the string are governed by the Polyakov action

$$
S_{\mathrm{P}}=-\frac{T_{0}}{2} \int d^{2} \sigma \sqrt{-\eta} \eta^{a b} G_{M N} \partial_{a} X^{M} \partial_{b} X^{N},
$$

where $\sigma_{1} \equiv \tau$ is a temporal worldsheet coordinate, $\sigma_{2} \equiv \sigma$ is a spatial worldsheet coordinate, $X^{M}(\tau, \sigma)=(t(\tau, \sigma), \vec{x}(\tau, \sigma), u(\tau, \sigma))$ are the string embedding functions that describe where the string is located within the spacetime metric that we are interested in probing, $\eta_{a b}$ is the worldsheet metric and $T_{0}=\frac{\sqrt{\lambda}}{2 \pi}$ is the string tension. We choose worldsheet coordinates such that the worldsheet metric takes the form

$$
\eta_{a b}=\left(\begin{array}{cc}
-\alpha(\tau, \sigma) & -1 \\
-1 & 0
\end{array}\right)
$$

with $\alpha(\tau, \sigma)$ being an arbitrary function. Just as we did in the spacetime metric (2.4), we have chosen worldsheet coordinates that are infalling Eddington-Finkelstein coordinates. Lines of constant $\tau$ are infalling null worldsheet geodesics. As we describe further below, we fix the function $\alpha(\tau, \sigma)$ by demanding $\sigma=u$.

Varying the Polyakov action with respect to the embedding functions $X^{M}$ yields dynamical equations of motion. Likewise, varying the Polyakov action with respect to the worldsheet metric yields a system of constraint equations. With our choice of infalling Eddington-Finkelstein bulk and worldsheet coordinates, the dynamical equations of motion take the simple form

$$
\dot{X}^{\prime N}+\Gamma_{A B}^{N} \dot{X}^{A} X^{\prime B}=0
$$

where $\Gamma_{A B}^{N}$ are Christoffel symbols associated with the metric (2.4) for the bulk spacetime and

$$
\begin{aligned}
\dot{X}^{M} & \equiv \partial_{\tau} X^{M}-\frac{1}{2} \alpha X^{M}, \\
X^{M} & \equiv \partial_{\sigma} X^{M} .
\end{aligned}
$$

The constraint equations are even simpler:

$$
\begin{aligned}
X^{\prime 2} & =0, \\
\dot{X}^{2} & =0 .
\end{aligned}
$$


The constraint equation (2.13a) is a temporal constraint. If (2.13a) is satisfied every where in space at one time, the dynamical equations (2.11) imply that it will be satisfied at all subsequent times. The constraint equation $(2.13 \mathrm{~b})$ is a boundary constraint. If $(2.13 \mathrm{~b})$ is satisfied at all times at one value of $\sigma$ the dynamical equations (2.11) imply it will be satisfied at all $\sigma$.

Given $X^{M}$ for all $\sigma$ at some value of $\tau$, the equations of motion (2.11) constitute a linear system of ordinary differential equations for $\dot{X}^{M}$. To solve these equations, in addition to specifying the initial conditions for $X^{M}$ one must specify five boundary conditions at all $\tau$ at the AdS boundary, where the string endpoint is located. Three boundary conditions are simply that the string endpoint moves on a given trajectory $\vec{x}_{o}(t)$

$$
\lim _{\sigma \rightarrow 0} \vec{x}(\tau, \sigma)=\vec{x}_{o}(t)
$$

We choose the trajectory

$$
\vec{x}_{o}(t)=\vec{\beta} t,
$$

for some constant velocity $\vec{\beta}$. These boundary conditions correspond to choosing to study a heavy quark, whose location after all coincides with the location of the endpoint of the string, that is moving with constant velocity $\vec{\beta}$ and that finds itself at $z=0$ at time $t=0$, meaning that this heavy quark finds itself right in the center of the collision that we wish to probe. We shall present results for several different values of $\vec{\beta}$.

Two more boundary conditions are required. For convenience, we choose

$$
\lim _{\sigma \rightarrow 0} t(\tau, \sigma)=\tau,
$$

so that the worldsheet time $\tau$ corresponds to the coordinate time $t$ at the boundary. The remaining boundary condition comes from demanding that the boundary constraint (2.13b) is satisfied at $\sigma=0$. The boundary conditions (2.15) and (2.16) and the boundary constraint $(2.13 \mathrm{~b})$ are satisfied at $\sigma=0$ provided that ${ }^{2}$

$$
\lim _{\sigma \rightarrow 0} \dot{t}=\frac{1}{2}\left[1+\frac{1}{\gamma}\right], \quad \lim _{\sigma \rightarrow 0} \dot{\vec{x}}=\frac{\vec{\beta}}{2}, \quad \lim _{\sigma \rightarrow 0} \dot{u}=-\frac{1}{2 \gamma},
$$

where $\gamma \equiv 1 / \sqrt{1-\vec{\beta}^{2}}$ as usual.

We can now see how to evolve the string forward in $\tau$, given an initial string profile specified by $X^{M}$ at some initial $\tau$. The algorithm has two steps. First we need to obtain $\dot{X}^{M}=(\dot{t}, \dot{\vec{x}}, \dot{u})$ at the initial $\tau$. We do this by observing that if we think of $\dot{X}^{M}$ as the dynamical variables, the equations of motion (2.11) are first order differential equations for $\dot{X}^{M}$ with the independent variable being $\sigma$. We can solve these equations for $\dot{X}^{M}$ as functions of $\sigma$ at the initial $\tau$ as long as we know $X^{\prime M}$ - which we do since we have been given $X^{M}$ for all $\sigma$ at the initial $\tau$ — and as long as we have boundary conditions for $\dot{X}^{M}$

\footnotetext{
${ }^{2}$ To see that this is true one can solve the string equations of motion (2.11) and the constraint equations (2.13) with a power series expansion in $\sigma$ near $\sigma=0$. In doing so one can directly incorporate the boundary conditions (2.15) and (2.16) into the series expansions. With the expansion known to order $\sigma$, one can easily see that the boundary limit of $\dot{X}^{M}$ takes the form (2.17).
} 
at $\sigma=0$ - which we have in (2.17). So, we solve (2.11) starting from $\sigma=0$ and obtain $\dot{X}^{M}$ at all $\sigma$ at the initial $\tau$. In the second step of the algorithm, we use (2.12a), rewritten as $\partial_{\tau} X^{M}=\dot{X}^{M}+\frac{1}{2} \alpha X^{M}$, to compute the field velocities $\partial_{\tau} X^{M}$. To do this we need the function $\alpha$. With the gauge choice $\sigma=u$, it is given simply by $\alpha=-2 \dot{u}$. With $\partial_{\tau} X^{M}$ known, we can then determine $X^{M}$ at the new $\tau$, thus completing the evolution of the system from the initial $\tau$ to a $\tau$ one time-step later. We then repeat.

All that we still need to specify is our choice of initial conditions for $X^{M}$ at an initial time $t$ that we shall typically take to be $-3 / \mu$, when the centers of the incident sheets of energy are $6 / \mu=12 w$ apart. In the distant past, well before the sheets of energy collide, the near-boundary geometry between the sheets of energy, which is where the heavy quark is located, is that of an equilibrium black brane with a small temperature $T_{\text {background }}$. This is so provided that we do not choose $|\vec{\beta}|$ so large that at our initial time the heavy quark is within one of the incident sheets. We shall make sure not to do this, which is to say that we shall make sure that at our initial time the heavy quark has not yet felt the sheets of energy that are soon going to hit it to any significant degree. We therefore choose initial conditions such that the string profile coincides with the trailing string solution of refs. $[1,2]$ moving at velocity $\vec{\beta}$, given in appendix A. Such initial conditions satisfy the temporal constraint equation (2.13a) near the AdS boundary. ${ }^{3}$

Constructing initial string profiles that are equilibrium solutions to the pre-collision geometry ensures that the future non-trivial evolution of the string is entirely due to the change in the bulk geometry associated with the collision event, not due to transients that would come along as artifacts of any other choice of initial conditions. However, as we shall describe below, although perturbing the initial string profile does result in an early-time transient before the collision happens, the change that results in the rate of energy loss of the heavy quark during and after the collision event is negligible. Because in an actual heavy ion collision a heavy quark is produced during the collision, we are not particularly interested in any aspect of the motion of our heavy quark before the collision of our sheets of energy. This, together with the insensitivity of the results during and after the collision that we are interested in to initial conditions and associated transients, means that our study could be repeated with other choices of initial conditions for the string without changes to our conclusions.

We discretize the $\sigma$ coordinate using pseudo-spectral methods. Specifically, we decompose the $\sigma$ dependence of all functions in terms of the first 20-35 Chebyshev polynomials.

\footnotetext{
${ }^{3}$ Our choice of initial conditions is slightly complicated by our choice of infalling Eddington-Finkelstein coordinates. In infalling Eddington-Finkelstein coordinates the bulk is the causal future of the boundary. At any given Eddington-Finkelstein time $t$, even in the distant past before the sheets of energy collide on the boundary, the gravitational shocks are colliding somewhere deep in the bulk. As a consequence of this, choosing the trailing string of refs. [1,2] will lead to a small violation of the temporal constraint equation (2.13a) deep in the bulk. However, as time progresses the violation of the constraint decreases in magnitude and then as time progress further towards $t=0$ when the sheets collide on the boundary, the portion of the string that violates the temporal constraint equation is rapidly enveloped by the event horizon of the black brane. Because of this, the initial violation of the temporal constraint equation is causally disconnected from physics near the boundary and hence is of no concern. We discuss this further in appendix $\mathrm{A}$.
} 
We then time evolve the string profile, typically from $t=-3 / \mu$ to $t=+6 / \mu$, according to the algorithm that we have described above using a fourth order Runge-Kutta ordinary differential equation solver.

\subsection{Extracting the drag force acting on the quark from the string profile}

Because the quark is a point-like source which is being pushed through the surrounding medium at a constant speed by some external agent, it must be transferring energy and momentum to the surrounding medium meaning that the boundary stress tensor is not conserved:

$$
\partial_{\mu} T^{\mu \nu}(x)=F^{\nu}(x)=-f^{\nu} \delta^{3}(\vec{x}-\vec{\beta} t),
$$

where $f^{\nu}$ is the four momentum lost by the quark per unit time. Note that, as always for a force, $f^{\nu}$ does not transform as a four-vector under Lorentz transformations. It is conventional to write

$$
\frac{d p^{\nu}}{d t}=f^{\nu}
$$

with $p^{\nu}$ the four momentum of the quark, even though in the setup we are analyzing the four momentum of the quark does not actually change. The external agent does work in order to move the quark at constant speed and, in so doing, transfers energy and momentum to the medium surrounding the quark; the quark does not slow down. If the external agent dragging the quark is a classical background electric field, the total stress tensor

$$
T_{\mathrm{tot}}^{\mu \nu}=T^{\mu \nu}+T_{\mathrm{EM}}^{\mu \nu},
$$

is conserved

$$
\partial_{\mu} T_{\mathrm{tot}}^{\mu \nu}=0
$$

Via the quark, energy and momentum flow from the electric field into the surrounding medium. The quantity $f^{\nu}$ is thus given by

$$
f^{\nu}(t)=-\int d^{3} x \partial_{\mu} T^{\mu \nu}(x)=\int d^{3} x \partial_{\mu} T_{\mathrm{em}}^{\mu \nu}(x) .
$$

Just as the near-boundary behavior of the metric encodes the expectation value of the boundary stress tensor, the near boundary behavior of the string profile encodes $f^{\nu}$. Specifically, $f^{\nu}$ can be identified as the flux of four momentum down the string [26]. The flux down the string can be extracted by noting that the total action for the holographic system,

$$
S_{\mathrm{tot}}=S_{\mathrm{P}}+S_{\mathrm{EM}}
$$

must be diffeomorphism invariant. The electromagnetic action $S_{\mathrm{EM}}$ only has support at the boundary and couples the string endpoint to the classical background electric field used to drag the quark (which is to say the string endpoint) at constant velocity.

Under an infinitesimal diffeomorphism $X^{M} \rightarrow X^{M}+\chi^{M}$ the variation in the Polyakov action (2.9) only has support at the boundary and reads

$$
\delta S_{\mathrm{P}}=\lim _{u \rightarrow 0} \int d^{4} x n_{N} \chi^{M} \sqrt{-G} \mathcal{T}_{M}^{N},
$$


where $D_{M}$ is the covariant derivative with respect to the bulk spacetime metric (2.4), $n_{N}$ is the normal to the boundary at $u=0$, and

$$
\mathcal{T}^{M N}(Y)=\frac{-T_{0}}{\sqrt{-G}} \int d^{2} \sigma \sqrt{-\eta} \eta^{a b} \partial_{a} X^{M} \partial_{b} X^{N} \delta^{5}(Y-X),
$$

is the string stress tensor. Likewise, the variation in the electromagnetic action is

$$
\delta S_{\mathrm{EM}}=\int d^{4} x \chi^{\mu} \partial_{\nu} T_{\mu \mathrm{EM}}^{\nu}=-\int d^{4} x \chi^{\mu} F_{\mu}
$$

where in the last line we used $\partial_{\nu} T_{\mu \mathrm{EM}}^{\nu}=-F_{\mu}$ with $F_{\mu}$ defined in (2.18). Upon demanding that the variation in the action vanish for all $\chi^{\mu}$, we conclude that

$$
F_{\mu}=\lim _{\mathrm{u} \rightarrow 0} n_{N} \sqrt{-G} \mathcal{T}_{\mu}^{N}
$$

from which the drag $f^{\mu}$ can easily be extracted via (2.18).

Setting the normal to the boundary $n_{M}=\delta_{M 5}$, one might naively conclude ${ }^{4}$ that $F_{\mu}=\lim _{\mathrm{u} \rightarrow 0} \sqrt{-G} \mathcal{T}_{\mu}^{5}$. However, this cannot be correct. Near the boundary some components of the string stress tensor diverge like $1 / u^{2}$. As a consequence of this divergence $\lim _{\mathrm{u} \rightarrow 0} \sqrt{-G} \mathcal{T}_{\mu}^{5}$ transforms nontrivially under the radial diffeomorphism (2.5). Simply put, the expression $\lim _{\mathrm{u} \rightarrow 0} \sqrt{-G} \mathcal{T}_{\mu}^{5}$ depends on one's choice of the gauge parameter $\xi$ used in solving Einstein's equations.

The unique remedy to the above problem is to set $n_{M}=\delta_{M 5}+u^{2} \partial_{M} \xi$. This choice is simply the near-boundary limit of $\delta_{5 N}$, transformed by (2.5). In other words, $n_{M}=$ $\delta_{M 5}$ would have been correct if we had used $\xi=0$ but is not correct once we make the transformation (2.5) that we needed to make in order to solve Einstein's equations. With the correct choice of $n_{M}, F_{\mu}$ in (2.27) is invariant under all infinitesimal diffeomorphisms, including those arising from eq. (2.5). We therefore conclude that

$$
F_{\mu}=\lim _{u \rightarrow 0}\left[\sqrt{-G}\left(\mathcal{T}_{\mu}^{5}+u^{2} \partial_{\alpha} \xi\left(\mathcal{T}_{\mu}^{\alpha}-\delta_{\mu}^{\alpha} \mathcal{T}_{5}^{5}\right)\right)\right]
$$

We note that $f^{\mu}$ can also be expressed in terms of the canonical worldsheet fluxes

$$
\pi_{M}^{a} \equiv \frac{\delta S_{P}}{\delta\left(\partial_{a} X^{M}\right)}=-T_{0} \sqrt{-\eta} G_{M N} \eta^{a b} \partial_{b} X^{N}
$$

A straightforward exercise using the string stress (2.25) and (2.29) shows that

$$
f_{\mu}=\lim _{u \rightarrow 0}\left[\pi_{\mu}^{\sigma}+u^{2} \pi_{5}^{\sigma} \partial_{\mu} \xi-u^{2} \pi_{\mu}^{a} \partial_{\alpha} \xi \partial_{a} X^{\alpha}\right]
$$

We see that for the choice $\xi=0$ the usual identification of force in terms of the canonical fluxes $[1,2]$ is reproduced.

\footnotetext{
${ }^{4}$ As we did, in a preliminary version of this study [27]. The drag forces calculated in ref. [27] are not correct but, comparing the figures from that preliminary report to those in section 3 where we present our results, the differences are not large.
} 


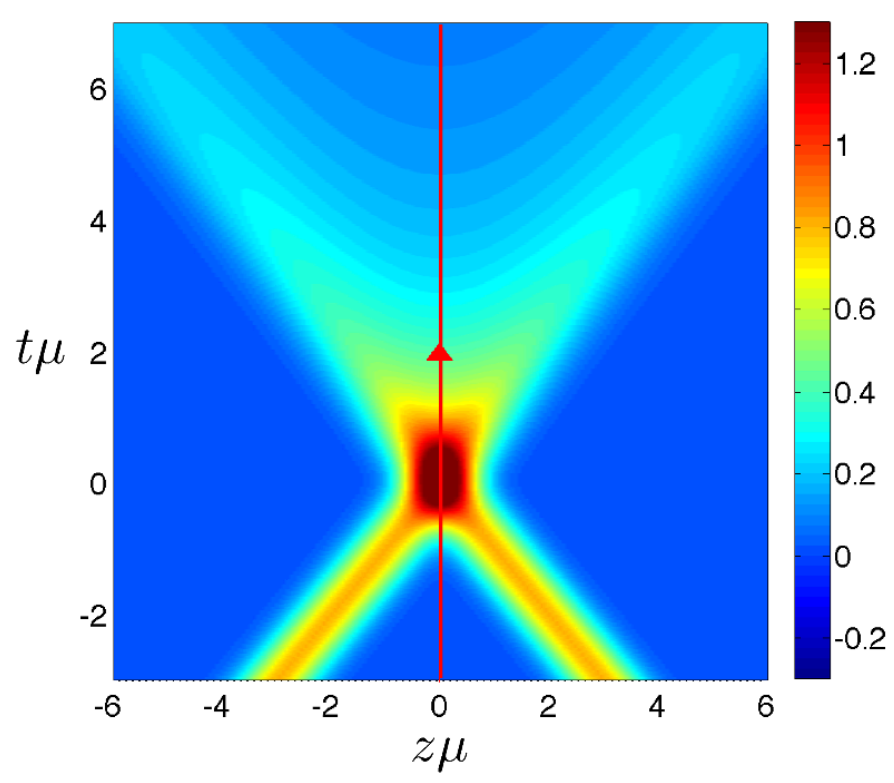

Figure 3. The path in the $(z, t)$-plane of a quark moving in the $z=0$ direction, i.e. with zero rapidity, is shown as the red line superimposed on a color-plot of the rescaled energy density $\mathcal{E}$, see eq. (2.6), in units of $\mu^{4}$. Thinking of the $x$-axis as perpendicular to the page, the quark is moving out of the page with velocity $\beta_{x}$.

\section{Results}

In this section we present and discuss the results that we have obtained for the drag force on a heavy quark being dragged through the colliding sheets of figure 1 . We shall initially choose the quark to be moving with a velocity $\vec{\beta}$ in the plane perpendicular to the direction of motion of the colliding sheets. Later we shall consider the more general case where the velocity of the quark has components both parallel to and perpendicular to the 'beam' direction. We shall compare our results to expectations based upon comparing the drag force that we calculated to what it would have been in a static homogeneous plasma with the same instantaneous energy density or parallel pressure or transverse pressure as that at the position of the quark. To further explore the effect of a time-dependent background on the drag force, in appendix B we consider a toy example in which the temperature increases with time but the plasma remains homogeneous and isotropic and does not undergo any expansion.

\subsection{Heavy quark with zero rapidity}

We begin with the case where the heavy quark is moving perpendicular to the 'beam' direction, which is to say perpendicular to the $z$-direction along which the sheets of energy collide. Although our heavy quark was present before the collision, from a phenomenological perspective this calculation may inform how we think about a heavy quark that is produced at $t=0$ in a heavy ion collision with zero rapidity moving with some perpendicular velocity $\vec{\beta}$. The $z=0$ path along which the heavy quark is moving is illustrated in 


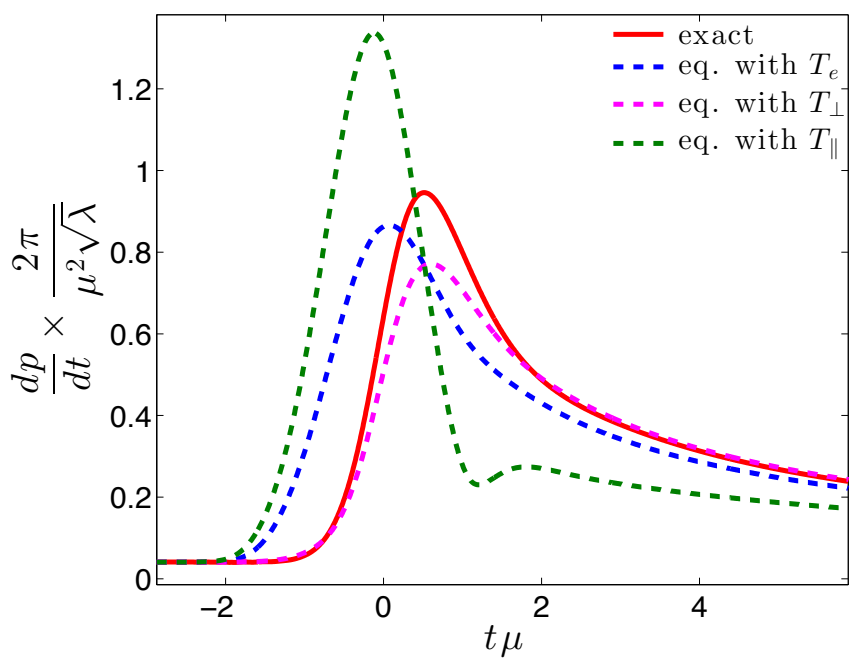

Figure 4. Drag force in units of $\mu^{2} \sqrt{\lambda} /(2 \pi)$ on a quark being pulled through the collision in the $x$-direction at $z=0$, i.e. at zero rapidity, with velocity $\beta_{x}=0.5$. The dashed curves show the drag force that the quark would experience in an equilibrium plasma with the same instantaneous energy density, parallel pressure or perpendicular pressure as that at the location of the quark. The dashed curves are described further in the text.

figure 3 , as is the energy density through which it moves. The parallel and perpendicular pressures of the material in which it finds itself are those that we plotted in figure 2 . The case of a quark moving at zero rapidity is simpler than the more general case that we will turn to next for two reasons: (i) a quark moving along the $z=0$ plane is always moving through fluid at rest, with $u^{\mu}=(1,0,0,0)$, meaning that in this case the local fluid rest frame is the same as the lab frame; (ii) the drag force acting on the quark with zero rapidity is directed antiparallel to the velocity vector, with no component of the force perpendicular to the direction of motion of the quark. We have calculated the drag force as described in section 2. Our results for quarks moving at zero rapidity with speed $\beta_{x}=0.5$ and $\beta_{x}=0.95$ are shown as the solid red curves in figures 4 and 5 , respectively.

We are interested in comparing our results to expectations based upon the classic result for the drag force required to move a heavy quark with constant velocity $\vec{\beta}$ through the equilibrium plasma in strongly coupled SYM theory, namely $[1,2]$

$$
\left.\frac{d \vec{p}}{d t}\right|_{\text {eq }}=\frac{\sqrt{\lambda}}{2 \pi}(\pi T)^{2} \frac{\vec{\beta}}{\sqrt{1-\beta^{2}}},
$$

where $T$ is the temperature of the equilibrium plasma. Out of equilibrium, the matter does not have a well-defined temperature. We can nevertheless use (3.1) to frame expectations for $d \vec{p} / d t$ at any point in spacetime, as follows. At $z=0$ the fluid is at rest, meaning that the lab frame in which we are working is the local fluid rest frame and the stress tensor for the fluid takes the form (2.8) which we now rewrite as

$$
T_{\text {rest frame }}^{\mu \nu}=\frac{\pi^{2} N_{c}^{2}}{8} \operatorname{diag}\left(3 T_{e}^{4}, T_{\perp}^{4}, T_{\perp}^{4}, T_{\|}^{4}\right) .
$$




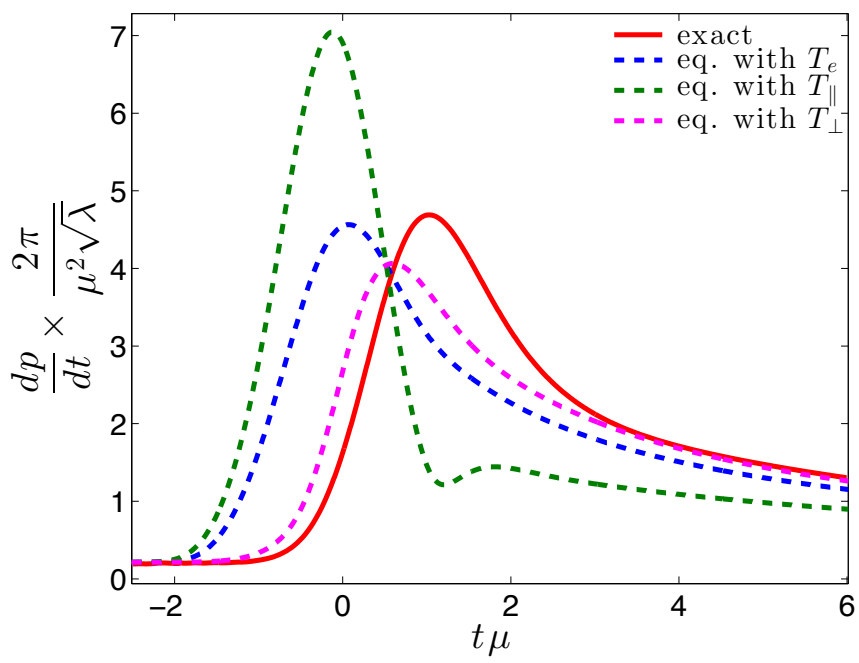

Figure 5. Same as figure 4 but for a quark moving at zero rapidity with a velocity $\beta_{x}=0.95$.

If the fluid were at rest in equilibrium, we would have $T_{e}=T_{\perp}=T_{\|}=T$. In the nonequilibrium setting of interest, through (3.2) we have defined three different "effective temperatures", no one of which is a true temperature since none can be defined. We can then use each of these three "temperatures" in the equilibrium expression (3.1), obtaining the three dashed curves in figures 4 and 5. Because the stress tensor is traceless, any one of the dashed curves can be obtained from the other two; only two are independent. This also explains why wherever two of the dashed curves cross the third must cross also. Note that none of the three dashed curves should be seen as a "prediction" for the actual drag force experienced by the heavy quark in the far-from-equilibrium conditions created by the collision of the sheets of energy. Rather, the three dashed curves tell us what the drag force would be in a static plasma with the same instantaneous energy density or transverse pressure or parallel pressure as that present in the far-from-equilibrium conditions at time $t$ and position $z=0$. The dashed curves are devices through which we use what we know about the drag force in an equilibrium plasma to frame expectations for the nonequilibrium case. $^{5}$

Comparing the drag force on the heavy quark, the solid red curves in figures 4 and 5 , to the three dashed curves yields many observations. Reading the figures from left to right, we first see that at very early times where $T_{e}=T_{\perp}=T_{\|}=T_{\text {background }}$ the drag force is indeed given by the equilibrium result (3.1) for a static plasma with temperature $T_{\text {background. }}$ Next, we see that the increase in the drag force due to the dramatic change

\footnotetext{
${ }^{5}$ Some support for this strategy is provided by analyses [28-30] of the drag force on a heavy quark moving through a fluid that is undergoing boost invariant expansion in one dimension and is translation invariant in the other two dimensions. In this setting, the gradient expansion of hydrodynamics becomes an expansion in powers of $1 / \tau^{2 / 3}$, with $\tau$ the proper time. (See, for example, refs. [11, 13].) At leading order, which is to say at late times, the fluid can be treated as ideal meaning that $T_{e}=T_{\perp}=T_{\|} \equiv T(\tau)$ with $T(\tau) \propto 1 / \tau^{1 / 3}$ [31]. In this approximation, the drag force on the heavy quark is indeed given by (3.1) with $T$ replaced by $T(\tau)$ [28, 29]. Ref. [30] includes an investigation of the next order corrections.
} 
in the stress tensor of the fluid corresponding to the collision of the sheets of energy is delayed. And, the delay in the increase of the drag force seems to increase with increasing heavy quark velocity. We shall return to this below. Third, we see that the peak value of the drag force is comparable to the expectations provided by the dashed curves, meaning that the peak value of the drag force in the far-from-equilibrium matter produced in the collision is not dramatically smaller or larger than what it would be in a static plasma with the same instantaneous energy density. These second and third observations suggest that a reasonable first-cut approach to modelling the drag on a heavy quark in a heavy ion collision would be to turn the drag force on roughly one "sheet thickness" in time after the collision and from then on use the equilibrium expression (3.1) with an effective temperature determined by the instantaneous energy density. Fourth, at late times when the expansion of the fluid is described well by viscous hydrodynamics we see in figures 4 and 5 that the drag force is best approximated by the value that it would have in a static plasma with the same instantaneous transverse pressure. To some degree this agreement is coincidental as we see that for $\beta=0.5$ our result is a little below (3.1) with the effective temperature $T_{\perp}$ while for $\beta=0.95$ our result is a little above this benchmark. What seems robust is the fact that at late times the actual force required to drag the quark through the expanding, cooling, anisotropic hydrodynamic fluid lies within the band of expectations spanned by the drag force in a static plasma with the same instantaneous energy density or parallel pressure or transverse pressure. For a heavy quark with zero rapidity, we see no qualitative deviation relative to these expectations, meaning that we see no qualitative consequences of the presence of gradients of the fluid velocity.

We are using initial conditions that correspond to a heavy quark which has been dragged through an equilibrium plasma with the low temperature $T_{\text {background }}$ for a long time before the collision. This, of course, is not reminiscent at all of what actually happens in a heavy ion collision, where the heavy quark is created during the collision. It is therefore important to check how sensitive our results for the drag force on the heavy quark during and after the collision are to the choices we are making for the initial shape of the string well before the collision. If our results during and after the collision were sensitive to our choice of initial conditions this would be problematic, since there can be no right answer to the question of what the initial conditions for a heavy quark before the collision should be since in actual heavy ion collisions there are no heavy quarks present then. Fortunately, as we illustrate in figure 6 we have found that our results of interest, namely our results for the drag force on the heavy quark during and after the collision, are quite insensitive to the choice of initial conditions. The solid red curve in figure 6 is the same as that in figure 4, while the blue, purple and green dashed curves correspond to initial string profiles chosen such that the initial drag force is zero, four or sixteen times that for the red curve. ${ }^{6}$ These perturbations to the initial shape of the string, which are in no way small, do have transient effects at early times but their effects on the drag force felt by the heavy quark during and after the collision are negligible.

\footnotetext{
${ }^{6}$ In obtaining these results, we have determined $t(\tau, \sigma)$ by solving the temporal constraint equation (2.13a) numerically; see appendix A for a discussion.
} 


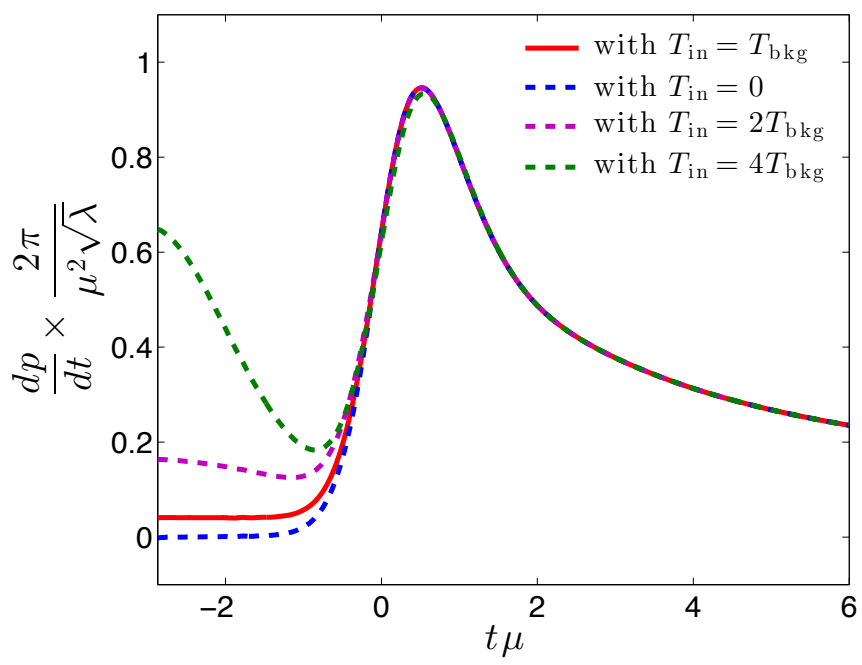

Figure 6. Illustration of how the drag force depends on the initial string shape. The four curves show the drag force on a quark whose string initially has the shape of the string trailing behind a heavy quark that is being dragged through an equilibrium plasma with temperatures ranging from zero to four times the actual temperature $T_{\text {background }}$ of the low temperature plasma present before the collision. The solid red curve is the same as the solid red curve in figure 4 . The other three curves show that by the time the collision occurs the drag force felt by the heavy quark is quite insensitive to the choice of initial conditions. Note that the initial drag force felt by the heavy quark varies between zero and sixteen times that for the red curve; the perturbations to the initial shape of the string illustrated here are not small, but their residual effects during and after the collision are. In all cases, the heavy quark is dragged with velocity $\beta_{x}=0.5$ and $\beta_{z}=0$.

Let us now return to the question of how the drag force changes with quark velocity $\beta$. Looking at figures 4 and 5 , the biggest effect of increasing $\beta$ is certainly the increase in the overall magnitude of the drag force. Since we have seen that our results are not dramatically different from the equilibrium expectations provided by the dashed curves in these figures, it is natural to look at the equilibrium formula (3.1) and ask to what degree the $\beta$-dependence in our results is described by assuming that the drag force scales with $\beta \gamma$. Figure 7 provides the answer. Almost all of the change in the overall magnitude of the drag force, e.g. the change in the height at which it peaks seen by comparing figures 4 and 5 , can be understood as scaling with $\beta \gamma$. The interesting $\beta$-dependent effect that remains in figure 7 is the time delay in the onset and peaking of the drag force. This time delay increases with increasing $\beta$. We investigate the $\beta$-dependence of the time delay in figure 8 . From this figure we see that a reasonable characterization of our results for the time at which the drag force peaks is that at low velocity it peaks about one sheet thickness after the time when (around the same time as) the drag force in an equilibrium plasma with the same with the same instantaneous energy density (transverse pressure) would peak while at higher velocities its peak is delayed by a time that increases approximately linearly with $\gamma$. In appendix B we investigate this time delay further by watching how the drag force responds in a background in which the temperature of the plasma is homogeneous in 


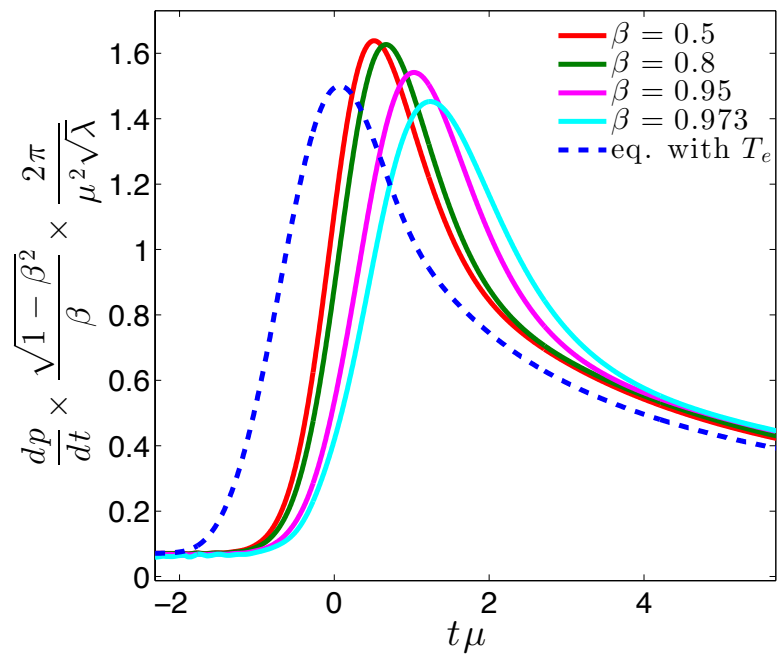

Figure 7. Rescaled drag force for four different quark velocities $\beta$, all in the $x$-direction as in figures 4 and 5 . We see that the largest $\beta$-dependence of our results in figures 4 and 5 can be understood by assuming that the drag force scales roughly with $\beta \gamma$. The remaining $\beta$-dependence seen here illustrates the fact that the delay in the onset of, and subsequent peak in, the drag force increases with $\beta$. The dashed curve shows the rescaled drag force that the quark would experience in an equilibrium plasma with the same instantaneous energy density. From (3.1) we see that, once we have rescaled by $\beta \gamma$, the dashed curve is $\beta$-independent.

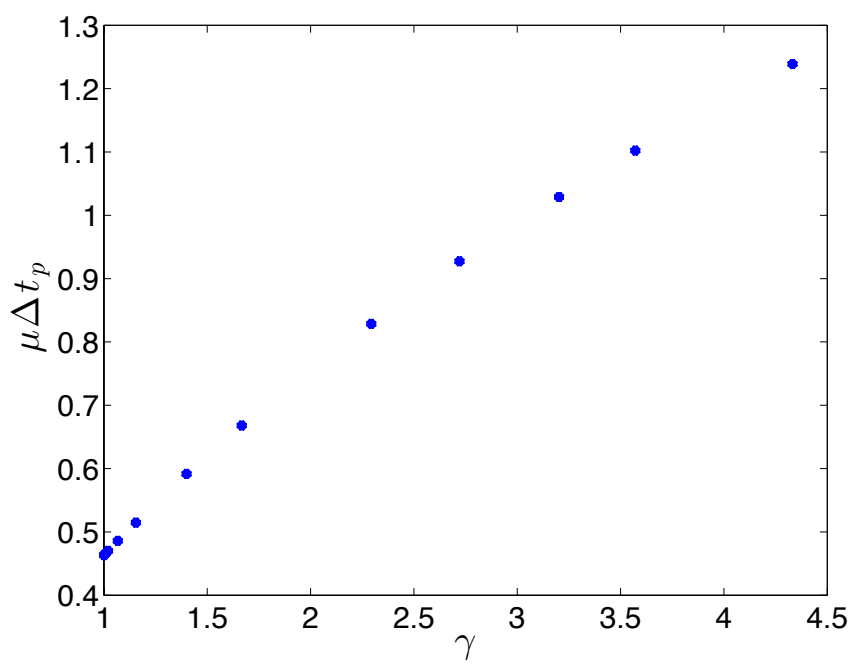

Figure 8. The time delay $\Delta t_{p}$ between the time at which the drag force on a heavy quark moving with velocity $\beta$ at zero rapidity peaks, i.e. the peak in the solid curve in figure 7 , and the time at which the dashed curve in figure 7 peaks. We plot $\Delta t_{p}$ vs. $\gamma$ for velocities ranging from $\beta=0.05$ to $\beta=0.973$. The increase in $\Delta t_{p}$ clearly has a component that is linear in $\gamma$, but the dependence is not completely linear. The value of $\Delta t_{p}$ at $\gamma \rightarrow 1$, i.e. at $\beta \rightarrow 0$, is similar to the delay between the peaks in the purple and blue dashed curves in figures 4 and 5 , which is $0.53 / \mu$. 
space but over some narrow range of time increases from a low value to a high value. This investigation indicates that the fact that the time delay increases roughly linearly with increasing $\gamma$ may be generic while the fact that (for the colliding sheets of energy above) we find that at low velocity the drag force peaks close to when the transverse pressure peaks is likely a coincidence. It also indicates that at low velocity the drag force may generically peak at a time that is about $1 /\left(\pi T_{\text {hydro }}\right)$ later than the time when the energy density peaks, where $T_{\text {hydro }}$ is the temperature defined from the fourth root of the energy density of the fluid at the time when the fluid hydrodynamizes. For the collision we are analyzing, this corresponds to a time delay of around one sheet thickness.

The analysis in appendix B supports a gravitationally intuitive picture in which a time delay of order $1 /\left(\pi T_{\text {hydro }}\right)$ corresponds to the time it takes information from deep inside the bulk, near the black hole horizon, to propagate to the boundary. The information that the geometry near the horizon has changed cannot propagate in the bulk faster than the speed of light, meaning that the time it takes for such a change to affect the string near the boundary - which is what determines the drag force on the heavy quark - is at least $\sim 1 /\left(\pi T_{\text {hydro }}\right)$. Furthermore, for a quark that is moving with a large $\gamma$ the length of the string that stretches from the quark at the boundary down to the near-horizon region is proportional to $\gamma$, suggesting that the time delay for a fast quark should include a contribution that is proportional to $\gamma /\left(\pi T_{\text {hydro }}\right)$. These considerations are somewhat heuristic, however, since the changes in the bulk geometry to which the string dragging behind the heavy quark responds do not occur only in the near-horizon region.

A time delay between a change in the stress tensor of the plasma and the resulting change in the drag force that is proportional to $\gamma$ can be understood qualitatively in terms of the boundary gauge theory as follows. Clearly, the drag force depends not only on the instantaneous stress tensor of the plasma in which the quark finds itself and the velocity of the quark but also on the history of the quark. In particular, our results suggest that the drag force takes on its "correct" value - i.e. the value it would have in an equilibrium plasma whose stress tensor is similar to the instantaneous stress tensor of the fluid around it — only if the fields that dress the moving quark are configured appropriately. Perhaps as the energy density of the plasma increases the longer wavelength fields that dress the moving quark must be stripped off leaving only those on length scales of order $1 / T_{e}$ and smaller. Perhaps when the energy density decreases those longer wavelength fields need to grow back. Whether or not such speculations are correct in detail, our results indicate that the drag force on the quark responds to changes in the conditions around the quark only after the fields carried along with the moving quark rearrange themselves in a way that takes some time. If the time this takes were constant in the rest frame of the quark, the time delay that we evaluate would be proportional to $\gamma \cdot{ }^{7}$

\subsection{Heavy quark with zero transverse momentum}

In this section and the next we shall consider more general cases in which the quark has some nonzero velocity $\beta_{z}$ in the 'beam' direction parallel to the direction of motion of the

\footnotetext{
${ }^{7} \mathrm{~A}$ time delay between some change in the environment in which a heavy quark finds itself and the resulting change in the drag force on the heavy quark has been seen in other contexts, see for example refs. $[32,33]$, in which it has also been attributed to the time it takes for the gluon fields around the moving quark to rearrange.
} 


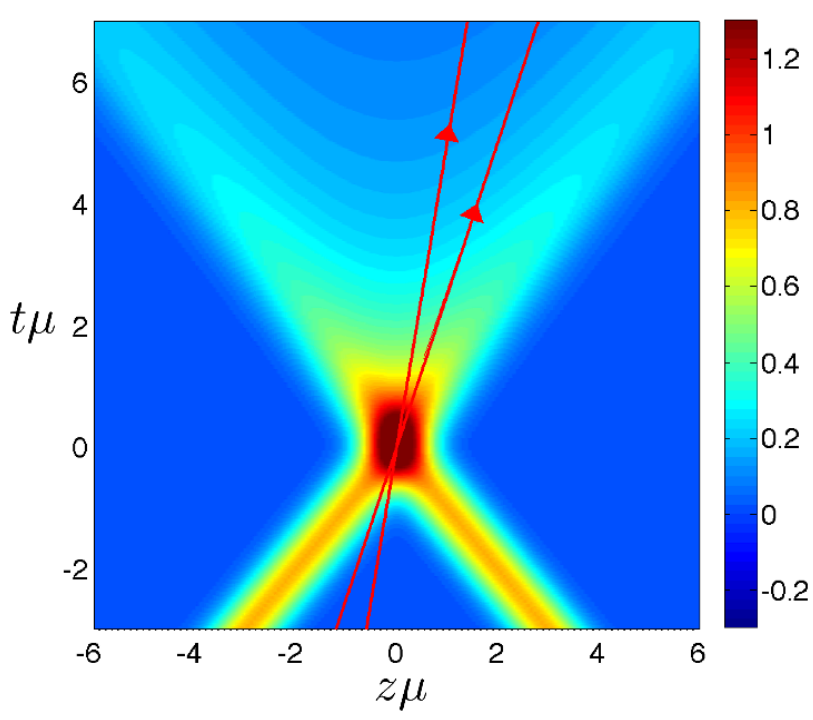

Figure 9. The red arrows show the trajectories in the $(z, t)$-plane of two quarks with nonzero rapidity, with $\beta_{z}=0.2$ and $\beta_{z}=0.4$. The trajectories are superimposed on the plot of the rescaled energy density $\mathcal{E}$ as in figure 3 . We shall consider cases in which the quark has zero or nonzero transverse velocity $\beta_{x}$. In all cases that we shall consider, the quark is pulled with constant velocity $\vec{\beta}$ through the energy density produced by the colliding sheets.

colliding sheets, which is to say that we shall allow the quark to have nonzero rapidity. As illustrated in figure 9 , we shall only consider trajectories in which the velocity $\vec{\beta}$ of the quark is constant and in which the initial position of the quark has been chosen such that at $t=0$ the quark is at $z=0$, meaning that the quark passes through the spacetime point at which the sheets of energy collide. Although in our setup the quark has existed for all time, we are of course interested in gaining qualitative insights into circumstances in which a heavy quark is produced at $z=t=0$ with some velocity $\vec{\beta}$. We shall not consider values of $\beta_{z}$ that are greater than 0.4 because we want to ensure that at the time $t=-3 / \mu$ at which we choose our initial conditions the quark has not yet felt the sheet of energy that is about to catch up with it in any significant away.

In order to obtain the greatest contrast with the case in which the quark has zero rapidity, analyzed above, in this section we shall consider the case in which the quark has a nonzero $\beta_{z}$ but has no transverse velocity, $\beta_{x}=0$. We shall allow both $\beta_{x}$ and $\beta_{z}$ to be nonzero in the next section.

The solid red curve in figure 10 shows the force on a quark with $\beta_{z}=0.4$ and $\beta_{x}=0$. To interpret the force shown in figure 10, we first need to ask what the velocity of the fluid is at the location of the quark as a function of time, since the drag on the quark should depend on its velocity relative to that of the moving fluid. The fluid velocity is always in the $z$-direction and its magnitude is given by

$$
\beta_{z}^{\text {fluid }} \equiv \frac{u^{z}}{u^{0}}
$$

where $u^{\mu}$ is the fluid velocity four-vector obtained from the stress tensor in the lab frame 


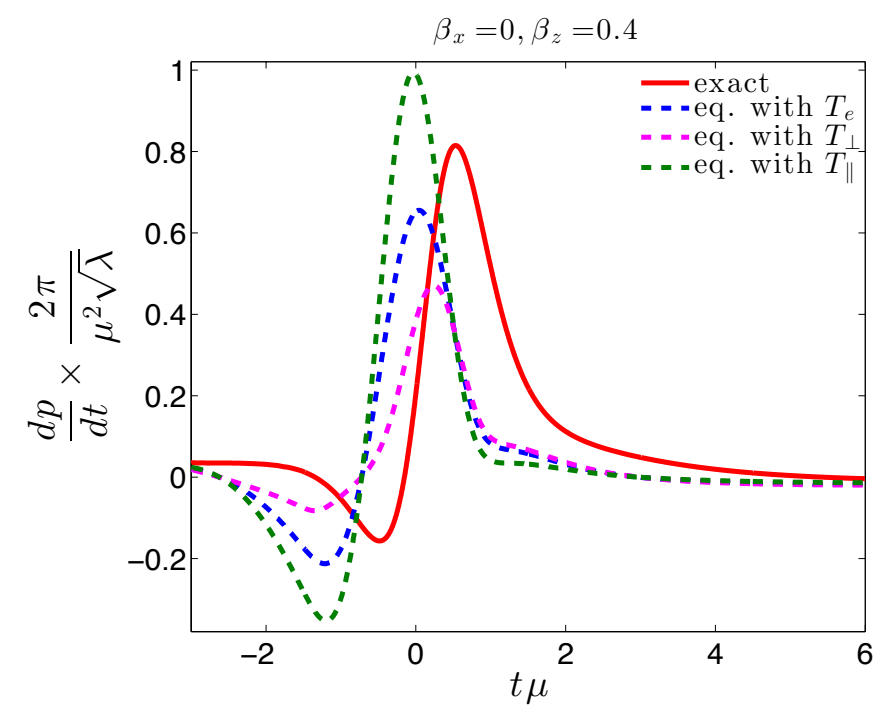

Figure 10. The 'drag' force (solid red curve) acting on a quark moving through the matter produced in the collision along a trajectory with $\beta_{z}=0.4$ and $\beta_{x}=0$. The force is shown in the lab frame. The dashed curves show what the force would be if in the local fluid rest frame it were given by the drag force that the quark would experience in an equilibrium plasma with the same instantaneous energy density, parallel pressure or perpendicular pressure as that in the local fluid rest frame at the location of the quark. The dashed curves are described further in the text.

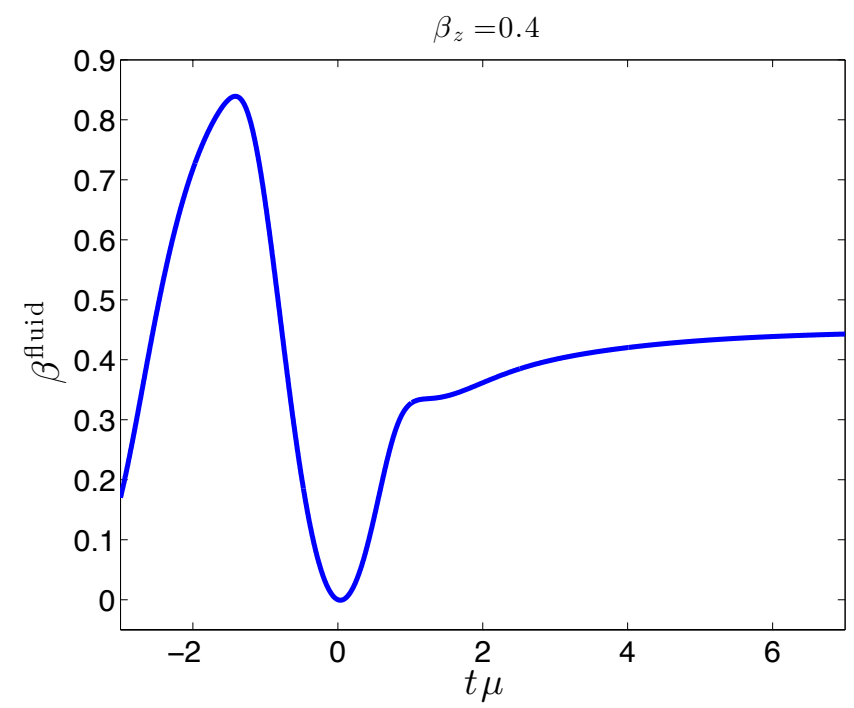

Figure 11. The fluid velocity $\beta^{\text {fluid }}$, defined in the text, at the location of a quark moving along the trajectory in figure 9 with $\beta_{z}=0.4$. If the hydrodynamic fluid at late times were boost invariant, $\beta^{\text {fluid }}$ would be given by $\beta_{z}=0.4$. Note that at late times $\beta^{\text {fluid }}>0.4$, meaning that the fluid is moving faster than the quark.

as described by eq. (2.7). We show $\beta^{\text {fluid }}$ in figure 11. Reading this figure from left to right while referring to figure 9: (i) We see that at the time $t=-3 / \mu$ at which we choose our initial conditions the quark is already feeling some effects of the fast-approaching sheet 
of energy. The quark is initially immersed in the background plasma, which is at rest, meaning that the fact that $\beta^{\text {fluid }} \neq 0$ can be attributed to the Gaussian tail of the sheet of energy which is approaching with velocity 1 . (ii) Then, the sheet of energy incident from the left catches up to the quark and as the energy of the sheet overwhelms that of the background plasma $\beta^{\text {fluid }}$ rises toward the speed of light. Note that the quantity $\beta^{\text {fluid }}$ can be computed as we have described even when the matter is far from equilibrium, as long as its energy density is positive [9]. At these early times $\beta^{\text {fluid }}$ should not really be thought of as the velocity of a fluid, although for convenience we shall refer to it as such. (iii) Next, the left-moving sheet of energy slams into the right-moving sheet and, briefly, $\beta^{\text {fluid }}$ is near zero. By symmetry, $\beta^{\text {fluid }}=0$ at all times at $z=0$, meaning that $\beta^{\text {fluid }}=0$ at the location of the quark at $t=0$. (iv) Finally, at late times after the fluid has hydrodynamized, $\beta^{\text {fluid }}$ is close to $\beta_{z}=0.4$. Note that if the hydrodynamic expansion at late times were boost invariant, the velocity of the fluid at late times on a trajectory with constant rapidity like the one that the quark is following would be given by the velocity of the quark itself. In other words, if the expanding fluid at late times were boost invariant, the curve in figure 11 would have $\beta^{\text {fluid }}=0.4$ at late times.

With an understanding of the velocity of the matter through which the heavy quark is moving now in hand, we can return to figure 10 and compare the drag force there with expectations based upon the force (3.1) on a heavy quark that is being dragged through plasma in equilibrium, as we did in figure 4. The expression (3.1) describes the force on a quark moving through matter at rest, so to use it at any given time $t$ we must first boost to a frame in which the matter through which the quark in figure 10 is moving is at rest at that time. That is, we boost by a velocity $\beta^{\text {fluid }}(t)$, plotted in figure 11 , to the local fluid rest frame. In the local fluid rest frame, the quark is moving in the $z$-direction with a velocity

$$
\beta_{z, \mathrm{RF}}=\frac{\beta_{z}-\beta^{\text {fluid }}}{1-\beta_{z} \beta^{\text {fluid }}}
$$

that can be positive or negative depending on whether $\beta^{\text {fluid }}$ is smaller or larger than the velocity $\beta_{z}$ of the quark in the lab frame. Next, we compute the stress tensor in the local fluid rest frame, where it takes the form (3.2), and use the temperatures $T_{e}, T_{\perp}$ and $T_{\|}$so obtained as well as the velocity $\beta_{z, \mathrm{RF}}$ from (3.4) in the expression (3.1). This gives us the drag force that the quark would experience if, in the local fluid rest frame, it were moving with velocity $\beta_{z, \mathrm{RF}}$ through an equilibrium plasma with the same instantaneous energy density, perpendicular pressure or parallel pressure as that of the matter at its location. Finally, we boost the three forces computed in this way back to the lab frame, using the appropriate Lorentz transformation for forces, given in the present context by ${ }^{8}$

$$
f_{\mathrm{eq}, \text { lab frame }}^{\mu}=\frac{1}{u^{0}+u^{z} \beta_{z, \mathrm{RF}}}\left(\begin{array}{c}
u^{0} f_{\mathrm{eq}, \mathrm{RF}}^{0}+u^{z} f_{\mathrm{eq}, \mathrm{RF}}^{z} \\
f_{\mathrm{eq}, \mathrm{RF}}^{x} \\
f_{\mathrm{eq}, \mathrm{RF}}^{y} \\
u^{0} f_{\mathrm{eq}, \mathrm{RF}}^{z}+u^{z} f_{\mathrm{eq}, \mathrm{RF}}^{0}
\end{array}\right),
$$

\footnotetext{
${ }^{8}$ It is a worthwhile check of the formalism for extracting the drag force from the bulk gravitational quantites that we have set out in section 2.3 to confirm that upon boosting the bulk black brane metric and the trailing string profile by a velocity $\beta^{\text {fluid }}$ and evaluating (2.30) one obtains (3.5).
} 
with $u^{\mu}$ the fluid four-velocity from eq. (2.7). We plot the three lab-frame forces computed as we have just described as the three dashed curves in figure 10. If we apply this algorithm in the more general case in which in the lab frame the quark is moving with both $\beta_{z}$ and $\beta_{x}$ nonzero, the result is

$$
\begin{aligned}
& f_{\text {eq, lab frame }}^{x}=\frac{\sqrt{\lambda}}{2 \pi}(\pi T)^{2} \gamma\left(u^{0} \beta_{x}-u^{z} \beta_{x} \beta_{z}\right) \\
& f_{\text {eq, lab frame }}^{z}=\frac{\sqrt{\lambda}}{2 \pi}(\pi T)^{2} \gamma\left(u^{0} \beta_{z}+u^{z}\left(\beta_{x}\right)^{2}-u^{z}\right),
\end{aligned}
$$

which can be written as

$$
f_{\text {eq, lab frame }}^{\mu}=-\frac{\sqrt{\lambda}}{2 \pi}(\pi T)^{2} \frac{1}{\gamma}\left(u_{\nu} u_{q}^{\nu} u_{q}^{\mu}+u^{\mu}\right),
$$

where we have defined $u_{q}^{\mu} \equiv \gamma(1, \vec{\beta})$ and have allowed for a generic choice of coordinate axes. The $T$ used in the expressions (3.6) or (3.7) can be either the $T_{e}$ or the $T_{\perp}$ or the $T_{\|}$ obtained from the stress tensor in the local fluid rest frame.

We can now compare the actual drag force on a quark moving in the $z$-direction, the solid red curve in figure 10, to the expectations for an equilibrium plasma moving with velocity $\beta^{\text {fluid }}$ with the same instantaneous energy density or pressure, shown as the dashed curves. In many respects, this comparison is as we found in figure 4 for a quark moving in the $x$-direction. As in figure 4, we see in figure 10 that the maximum value of the drag force in the far-from-equilibrium matter produced during the collision is within the expectations for the maximum drag force spanned by the three dashed curves. And, as in figure 4, we see a time delay in the rise in the magnitude of the drag force. Here, though, the drag force first goes negative as the heavy quark is hit from the left by the sheet of energy going in the same direction and only then goes positive as the second sheet of energy slams into the first.

The most interesting differences between figures 10 and 4 arise at late times. First, we see that the magnitude of the drag force at late times is much smaller in figure 10 than in figure 4. This reflects the fact that at late times the quark and the fluid are moving at comparable velocities, see figure 11 , meaning that their relative velocity is small. In fact, at $t \simeq 2.8 / \mu$ the fluid velocity and the quark velocity are equal - the dashed curves in figure 10 therefore cross zero there. Interestingly, we see that the solid curve stays positive for quite a long time afterwards until $t=5.0 / \mu$ meaning that there is an extended period of time when: (i) The fluid has hydrodynamized. (ii) The fluid is moving faster than the quark, which would suggest that the 'drag' force on the quark needed to keep it moving at constant $\beta_{z}$ should be a force pulling backward on it, toward negative $z$, pulling against the push from the fluid that is moving faster than the quark. That is, we expect that $d p / d t$ should be negative, as is indeed the case for the dashed curves in figure 10. (iii) Instead, the quark is still being dragged forward, toward positive $z$, with $d p / d t$ positive. This means that in the local fluid rest frame the force that the external agent must exert in order to move the quark towards the left acts toward the right. The quark is moving towards the left in this frame but the force exerted on it by the liquid 


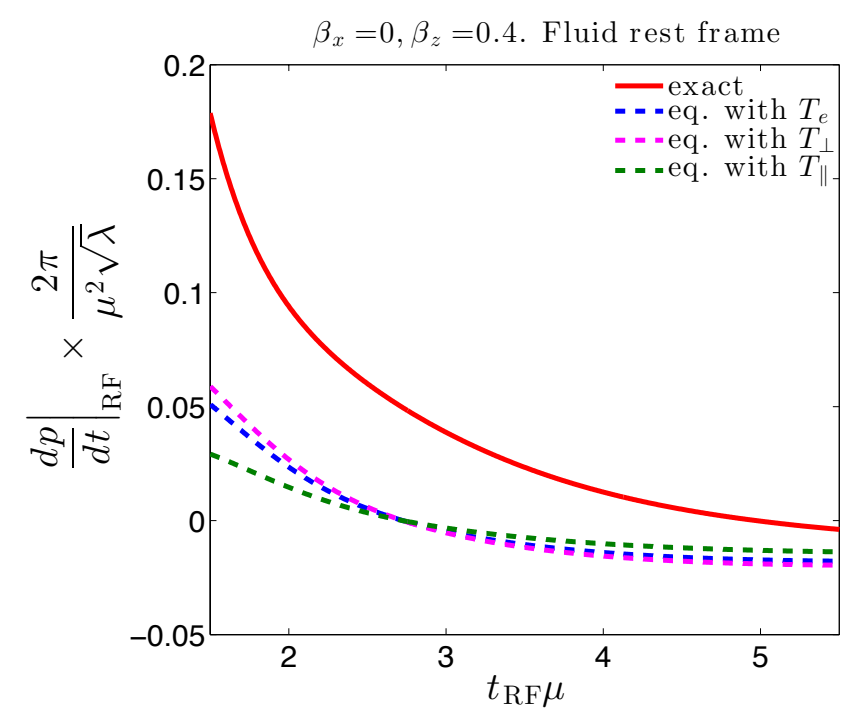

Figure 12. Forces as in figure 10, plotted here in the local fluid rest frame and focusing on times $t_{\mathrm{RF}}>1.5 / \mu$ to better illustrate the behavior after hydrodynamization. After $t_{\mathrm{RF}}=2.8 / \mu$, the quark is moving to the left in the local fluid rest frame (in the lab frame, the fluid is moving faster than the quark) and the dashed curves accordingly lead us to expect that the force needed to drag the quark leftward in the local fluid rest frame should be a force acting toward the left. Instead, we see that between $t_{\mathrm{RF}}=2.8 / \mu$ and $t_{\mathrm{RF}}=5.0 / \mu$, the 'drag' force that must be exerted to maintain the leftward motion acts toward the right!

through which it is moving is also acting toward the left, pushing it in the direction of its motion rather than dragging it in the opposite direction! We illustrate this in figure 12 by plotting the force exerted on the quark in the local fluid rest frame. The reason that a result as surprising as this is possible is that in formulating our expectations, as shown via the dashed curves, we are completely neglecting the effects of spatial gradients in the fluid velocity. ${ }^{9}$ Unlike in figure 4 , in figure 10 these gradients are aligned parallel to the direction in which the quark is moving; the lesson we learn is that in this circumstance the gradients in the fluid velocity can have qualitative effects on the 'drag' force that must be exerted on the quark. Qualitative effects of the gradient in the fluid velocity arise in figures 10 and 12 but not in figure 4 both because the effects of the gradient on the 'drag' force are larger in absolute magnitude when the gradient is aligned with the motion of the quark and because in this case the drag force in the absence of gradients would be very small since the relative velocity of the quark and the fluid is so small.

The red curves in figure 13 show the gradient of the fluid velocity in the lab frame and in the local fluid rest frame, in each case projected onto the quark velocity in that frame,

\footnotetext{
${ }^{9}$ We have also neglected spatial gradients in the energy density of the fluid. In the following, we will show that after hydrodynamization the effects of spatial gradients on the drag force that we compute correlate well with the behavior of the gradients of the fluid velocity. We have checked that the spatial gradients of the energy density are small at these late times, as are their effects on the force that we compute.
} 

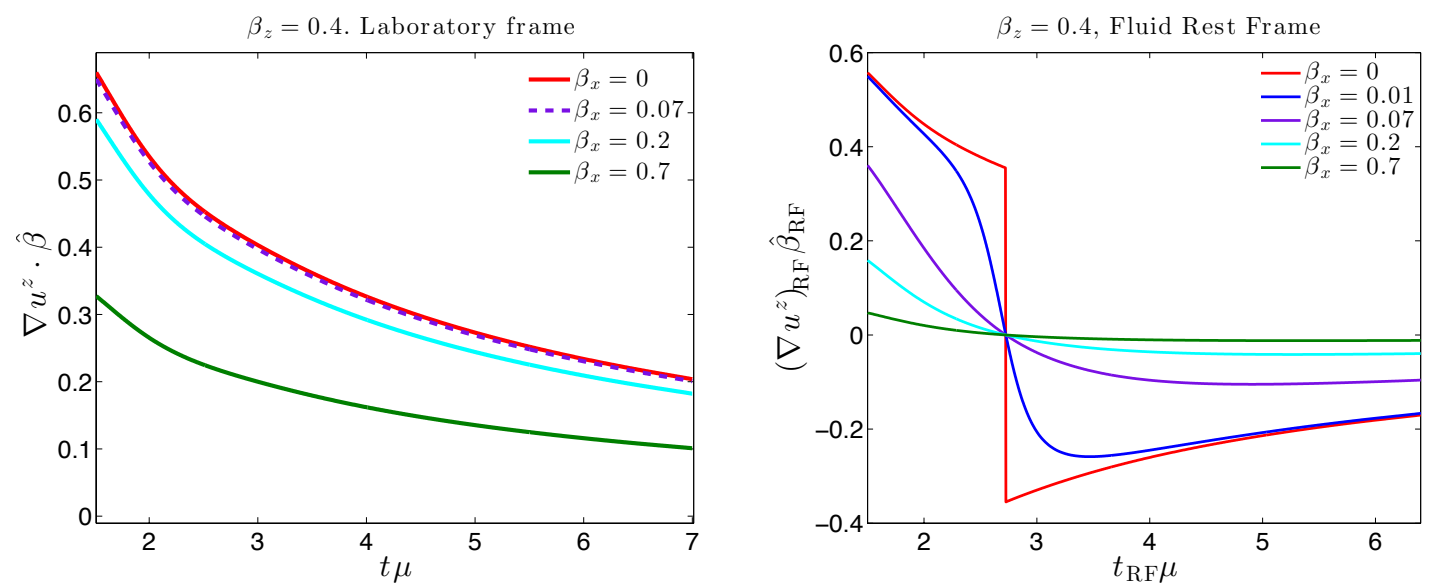

Figure 13. Component of the gradient of the fluid velocity in the direction of motion of the quark at the location of the quark in the lab frame (left panel) and in the local fluid rest frame (right panel). The differences between the two panels arises because $\beta_{z, \mathrm{RF}} \ll \beta_{z}$. In all curves in both panels, the trajectory of the quark has $\beta_{z}=0.4$. The different curves are for trajectories with varying values of $\beta_{x}$. At $t_{\mathrm{RF}}=2.8 / \mu$, the sign of $\beta_{z, \mathrm{RF}}$ flips, which is to say that in the lab frame the velocity of the fluid exceeds the velocity of the quark. We see in the right panel that adding a $\beta_{x}$ that is quite small compared to $\beta_{z}$ in the lab frame, but is comparable to $\beta_{z, \mathrm{RF}}$, significantly reduces $\left(\nabla u^{z}\right)_{\mathrm{RF}} \cdot \hat{\beta}_{\mathrm{RF}}$.

namely

$$
\left(\nabla u^{z}\right) \cdot \hat{\beta} \equiv \frac{\partial u^{z}}{\partial z} \frac{\beta_{z}}{\beta}
$$

and

$$
\left.\left(\nabla u^{z}\right)_{\mathrm{RF}} \cdot \hat{\beta}_{\mathrm{RF}} \equiv \frac{\partial u^{z}}{\partial z}\right|_{\mathrm{RF}} \frac{\beta_{z, \mathrm{RF}}}{\beta_{\mathrm{RF}}}
$$

for the trajectory with $\beta_{z}=0.4$ and $\beta_{x}=0$. For trajectories with $\beta_{z}=0$ and $\beta_{x}>0$, both quantities vanish identically. Figure 13 shows that if we start with $\beta_{z}=0.4$ and add a nonzero $\beta_{x}$ that is small compared to $\beta_{z}$ in the lab frame but that is large compared to $\left|\beta_{z, \mathrm{RF}}\right|$, for example $\beta_{x}=0.2$, the quantity $\left(\nabla u^{z}\right)_{\mathrm{RF}} \cdot \hat{\beta}_{\mathrm{RF}}$ is substantially reduced while in the lab frame $\left(\nabla u^{z}\right) \cdot \hat{\beta}$ is not much changed. This suggests that if we analyze the drag force on a quark that follows a trajectory with $\beta_{z}=0.4$ and $\beta_{x}=0.2$, we should find results that in the local fluid rest frame are more similar to those in figure 4 but in the lab frame are more similar to those in figure 10. We shall confirm this expectation in the next section, but in so doing we shall discover a second surprise.

\subsection{Heavy quark with nonzero rapidity and transverse momentum}

In this section we analyze the force that must be exerted in order to move a heavy quark through the colliding sheets of energy along a trajectory with nonzero $\beta_{x}$ and $\beta_{z}$, which is to say with both transverse momentum and rapidity nonzero. We start by considering a trajectory with $\beta_{z}=0.4$ as in figures 10 and 11 but now with $\beta_{x}=0.2$. Because the velocity of the quark now has a component perpendicular to the velocity of the fluid at the location of the quark (this was not the case in both previous sections) we now expect and 


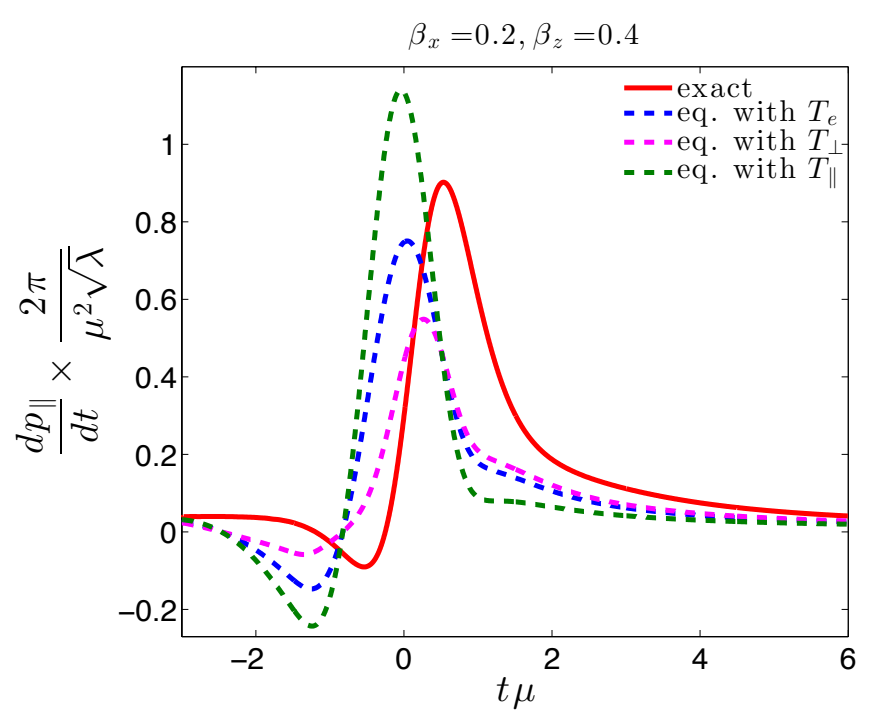

Figure 14. The force (solid red line) in the lab frame that must be exerted in the direction of motion of the quark (in the lab frame) in order to drag it with a velocity $\beta_{x}=0.2$ and $\beta_{z}=0.4$, a trajectory with nonzero transverse momentum and rapidity. The dashed curves, computed as described in the previous section, show what this force would be if in the local fluid rest frame the quark were being dragged through a plasma with the same instantaneous energy density, perpendicular pressure or parallel pressure as that at the location of the quark.

find that the force that must be exerted in order to move the quark along this trajectory has a component perpendicular to the direction of motion of the quark.

Let us first look at the drag force parallel to the direction of motion the quark in the lab frame,

$$
\frac{d p_{\|}}{d t} \equiv \frac{\vec{f} \cdot \vec{\beta}}{\beta},
$$

which is shown as the solid red curve in figure 14. (Note that $d p_{\|} / d t$ and, below, $d p_{\perp} / d t$ refer to the force parallel and perpendicular to the direction of motion of the quark; in contrast, $T_{\|}$and $T_{\perp}$ are defined via the pressures in the fluid that act in the directions parallel to and perpendicular to the direction of motion of the colliding sheets and hence of the fluid, i.e. the $z$-direction.) We see that, as in both sections 3.1 and 3.2, the dashed curves provide a reasonable guide to the peak value of the drag force but the actual force peaks later than the dashed curves do, a time delay that is by now familiar.

At late times, after the fluid has hydrodynamized, we see behavior that is more similar to that in section 3.2 in the sense that the drag force in the direction of motion of the quark is affected by the gradients in the fluid velocity to such a degree that it is well outside the expectations spanned by the three dashed curves. Unlike when $\beta_{x}$ was zero, though, with $\beta_{x}=0.2$ at least the sign of the force is the same for the solid and dashed curves. The dashed curves nevertheless fail to give a qualitative description of the actual drag force after the fluid has hydrodynamized. This is consistent with the left panel of figure 13 which shows that, when $\beta_{z}=0.4$, turning on $\beta_{x}=0.2$ does not substantially reduce the magnitude of the component of the gradient of the fluid velocity in the direction of motion 


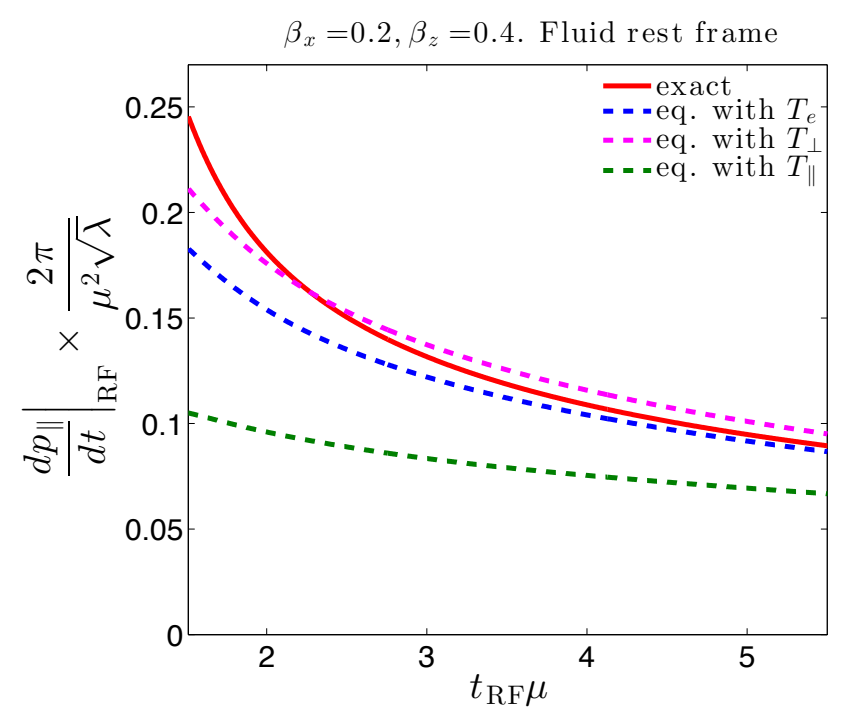

Figure 15. Forces as in figure 14 plotted here in the local fluid rest frame and focusing on times $t_{\mathrm{RF}}>1.5 / \mu$ to better illustrate the behavior after hydrodynamization. We saw in figure 12 that when $\beta_{z}=0.4$ and $\beta_{x}=0$ the dashed curves that show what the drag force in the local fluid rest frame would be in a spatially homogeneous plasma with the same instantaneous energy density or transverse pressure or parallel pressure failed to describe the actual force on the quark, because they neglected the effects of gradients in the fluid velocity on the force. Here, with $\beta_{x}=0.2$, we see that after hydrodynamization the actual force on the quark falls within the range of expectations spanned by the dashed curves.

of the quark - in the lab frame. In figure 15 we see that the story is different in the local fluid rest frame. With $\beta_{x}=0.2$, we find that the drag force in this frame does lie within the range of expectations spanned by the three dashed curves. This is consistent with the right panel of figure 13 , where we saw that, because $\left|\beta_{z, \mathrm{RF}}\right| \ll \beta_{z}$, when we turn on $\beta_{x}=0.2$ we do substantially reduce the component of the gradient of the fluid velocity in the direction of motion of the quark - in the local fluid rest frame.

Turning now to the force acting perpendicular to the direction of motion of the quark, we fix our sign conventions by defining this as

$$
\frac{d p_{\perp}}{d t} \equiv f^{z} \frac{\beta_{x}}{\beta}-f^{x} \frac{\beta_{z}}{\beta} .
$$

It is plotted as the solid red curve in the left panel of figure 16. The dashed curves show what the force perpendicular to the direction of motion of the quark would be in the lab frame if, in the local fluid rest frame, the quark were moving through an equilibrium plasma with the same instantaneous energy density, perpendicular pressure, or parallel pressure as that at the location of the quarks.

The force perpendicular to the direction of motion of the quark is a new development, present only when both $\beta_{x}$ and $\beta_{z}$ are nonzero. In the lab frame, the existence of a perpendicular force is no surprise since in this frame of reference the quark is moving through a fluid whose velocity includes a component perpendicular to its own. For this reason, in the left panel of figure 16 the dashed curves show that even if there were no 

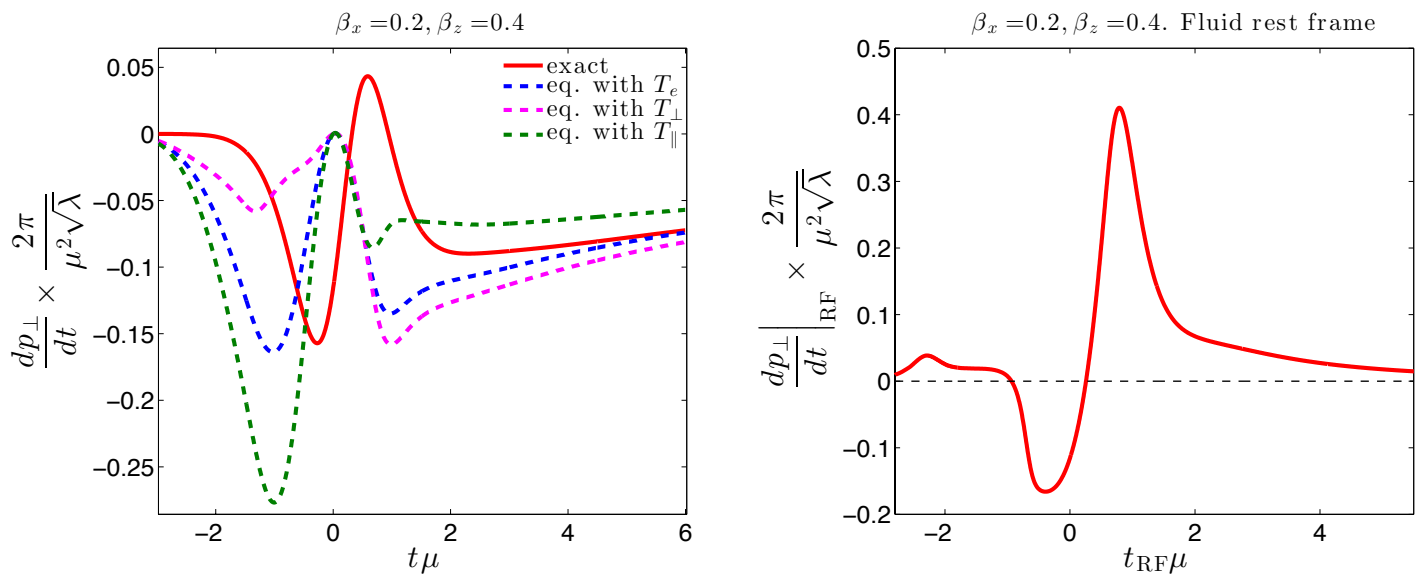

Figure 16. Left panel: as in figure 14, but here we plot the force that must be exerted perpendicular to the direction of motion of the quark, in the lab frame. Note the scale on the vertical axis: this component of the force is significantly smaller than the component of the force in figure 14 . Right panel: the force that must be exerted perpendicular to the direction of motion of the quark in the local fluid rest frame. If, in this frame, the quark were moving through a homogeneous fluid (e.g. the equilibrium plasma with the same instantaneous energy density or pressure as that at the location of the quark) the force in this frame could only act parallel to the direction of the quark. The dashed curves therefore all vanish in the right panel. The actual force does not vanish and is in fact quite substantial at its peak: even in the local fluid rest frame, the force needed to drag the quark includes a component perpendicular to its direction of motion.

velocity gradients in the fluid the force would include a component perpendicular to the direction of motion of the quark in the lab frame. This poses an obvious question: boost to the local fluid rest frame and ask whether in that frame the drag force is parallel to the direction of motion of the quark. We show in the right panel of figure 16 that the answer is no: even in a reference frame in which at each point in time we have boosted to a frame in which the quark is moving through a fluid at rest, the force required to drag the quark along its trajectory through this fluid includes a component perpendicular to the direction of motion of the quark! In this frame, in the absence of gradients in the fluid there could be no component of the force perpendicular to the motion of the quark. The right panel of figure 16 thus represents a stark consequence of the presence of spatial gradients in the matter produced in the collision. The effect is largest at early times, when the matter is far from equilibrium. At these early times, the effect is large indeed: the peak value of the force perpendicular to the motion of the quark is about half as large as the peak value of the drag force that acts parallel to the velocity of the quark. The effect is also nonvanishing at late times, after the expanding fluid has hydrodynamized. We find this result to be generic, arising for any trajectory in which both $\beta_{x}$ and $\beta_{z}$ are nonzero. A drag force that includes a component perpendicular to the direction of motion of the quark through a medium arises in other contexts in which some anisotropy in the medium has been introduced [34-36]. Here we see this phenomenon arising as a robust consequence of gradients in the fluid velocity - a form of anisotropy that must be present in heavy ion collisions. 

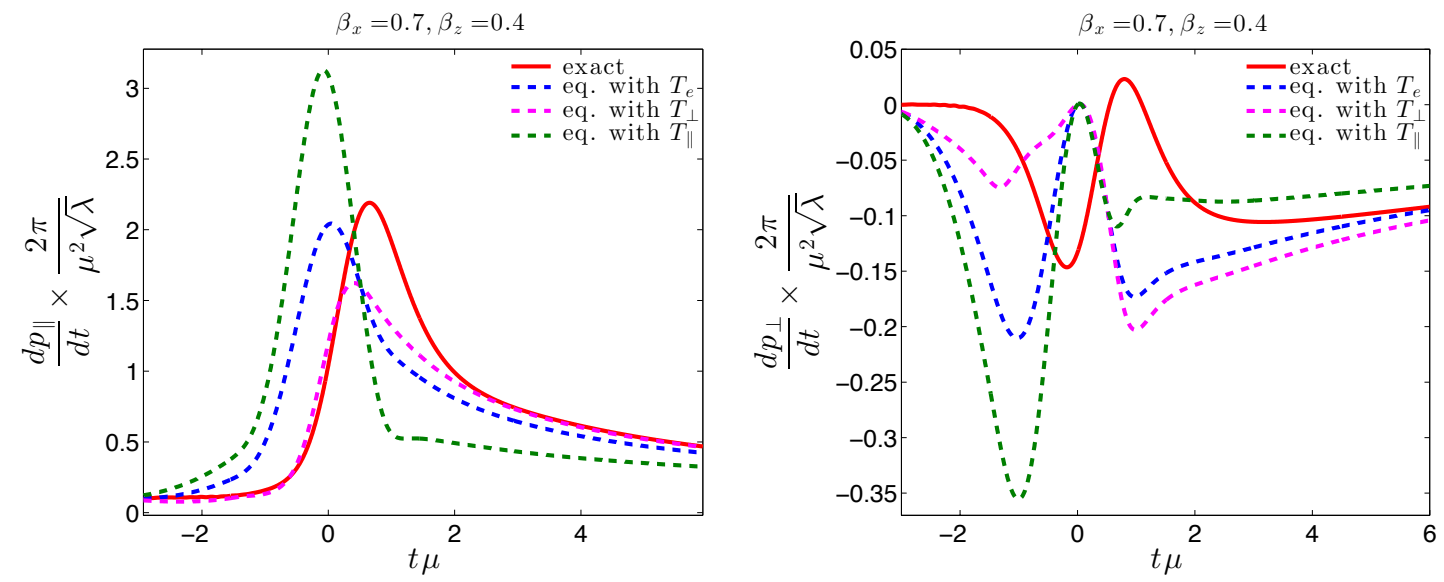

Figure 17. Drag force parallel to (left panel) and perpendicular to (right panel) the direction of motion of the quark in the lab frame, as in figure 14 and the left panel of figure 16, but here for a quark with $\beta_{x}=0.7$ and $\beta_{z}=0.4$.
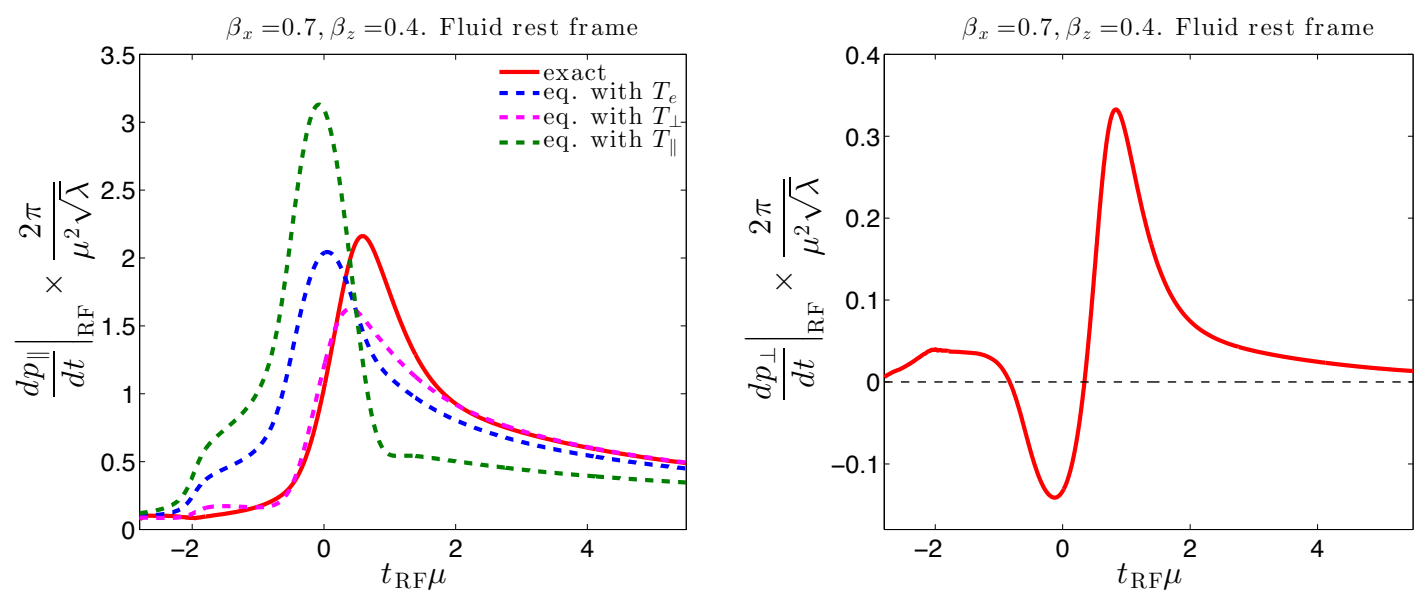

Figure 18. As in figure 17, but here in the local fluid rest frame.

To get a sense of how generic our results are, we close this section by illustrating them for the case where the heavy quark is dragged along a trajectory with $\beta_{x}=0.7$ and $\beta_{z}=0.4$. In figure 17 we first show our results in the lab frame, finding results that are similar in most respects to those at smaller $\beta_{x}$ that we saw in figures 14 and 16 . The important distinction is that in figure 17 we see that at late times, after hydrodynamization, the drag force that we calculate is well described by the dashed curves meaning that, unlike in figure 14, here there are no qualitative effects of the gradients in the fluid velocity apparent. We can understand this by noting from the left panel of figure 13 that by increasing $\beta_{x}$ from 0.2 to 0.7 we have substantially reduced the component of the gradient of the fluid velocity in the direction of motion of the quark in the lab frame.

In figure 18, we show our results for the case with $\beta_{x}=0.7$ and $\beta_{z}=0.4$ after boosting to the local fluid rest frame. Again, we find that even in a frame in which the quark is moving through a fluid that is instantaneously at rest the force required to drag the quark 
along its trajectory includes a component perpendicular to the trajectory. We see that this effect of gradients in the fluid velocity is somewhat smaller in absolute terms, and much smaller relative to the drag force in the direction of motion of the quark, here where $\beta_{x}=0.7$ than it was in the right panel of figure 16 , where $\beta_{x}=0.2$. This reflects the fact that as $\beta_{x}$ increases at fixed $\beta_{z}$ the component of the gradient of the fluid velocity in the direction of motion of the quark decreases.

\section{Conclusions and lessons for heavy ion physics}

The straightforward approach to modelling the rate of energy loss of heavy quarks produced in a heavy ion collision proceeds as follows: (i) Use the equilibrium equation of state to turn the proper energy density as a function of space and time in the collision (for example described via viscous hydrodynamics) into an effective temperature as a function of space and time; (ii) Use perturbative QCD to calculate the distribution of the initial positions and momenta of heavy quarks produced via hard scattering at the earliest moments of the heavy ion collision; (iii) Use the effective temperature from (i) in the expression (3.1) for the drag force in a homogeneous plasma in thermal equilibrium, perhaps with the overall prefactor in (3.1) turned into a parameter to be fit to data; (iv) Use the resulting spacetimedependent drag force, and consequent energy loss rate, in a Langevin equation employed to model the dynamics of heavy quarks in heavy ion collisions, as for example in refs. [37-42].

Our results indicate that a straightforward approach along the lines above can reasonably be applied even at very early times, before hydrodynamics applies. In particular, even though the peak value of the energy loss in the matter produced in the collision of the two sheets of energy that we have analyzed occurs before hydrodynamization, at a time when the matter produced in the collision is still far from equilibrium, this peak value is nevertheless reasonably well reproduced by the straightforward approach. Certainly there is no sign of any significant "extra" energy loss arising by virtue of being far from equilibrium. The message of our calculation seems to be that if we want to use (3.1) to learn about heavy quark energy loss in heavy ion collisions, it is reasonable to apply it throughout the collision, even before equilibration, defining the $T$ that appears in it through the energy density. The error that one would make by treating the far-from-equilibrium energy loss in this way is likely to be smaller than other uncertainties. We anticipate that this is the most robust lesson for heavy ion physics that we can draw from our results.

It also seems to be a generic feature of our results that there is some time delay after the collision before the rate of energy loss of the heavy quark rises to its peak value, even though it is during this very earliest time that the matter in which the quark is immersed has the very highest energy density. Although we have not characterized the delay time in the case of the collision of sheets of energy quantitatively, our analysis of this delay in a simpler setting suggests that it is of order $1 /\left(\pi T_{\text {hydro }}\right)$ (where $T_{\text {hydro }}$ is the temperature of the fluid when it hydrodynamizes) for a heavy quark whose velocity $\beta$ through the matter is not relativistic, and increases slowly as $1 / \sqrt{1-\beta^{2}}$ increases. This would correspond to a delay of something like $0.1-0.2 \mathrm{fm} / c$ in a heavy ion collision at RHIC. This delay suggests that it takes a little time after the heavy quark is enveloped by matter with a high 
energy density before the gluon fields around the heavy quark respond to the presence of the matter, with the drag and energy loss rising only after this response. Although our strongly coupled calculation cannot provide a complete characterization of the very earliest moments of a heavy ion collision, it is possible that this qualitative lesson may carry over. It would be interesting to use a model of heavy quark dynamics in heavy ion collisions to investigate whether a time delay along these lines has observable consequences.

The straightforward approach to modelling the drag force on a heavy quark in a heavy ion collision is built upon the result (3.1) for a homogeneous fluid and therefore neglects all effects of gradients in the fluid velocity. A third qualitative lesson that we can infer from our results is that this neglect works reasonably well for heavy quarks with small rapidity, whose velocities are close to perpendicular to the gradient in the fluid velocity.

We have found qualitative consequences for the drag force on heavy quarks with larger rapidity, moving closer to parallel to the gradient in the fluid velocity, arising from the presence of a velocity gradient, which is to say qualitative phenomena that are not present at all in the straightforward approach that we have sketched above. For example, we have found that because of the gradients in the fluid the force exerted by the fluid on a heavy quark that has a small velocity relative to the fluid at its location can sometimes point in the same direction as the velocity of the quark, rather than dragging on it. And, generically, we find that the force exerted by the fluid on a heavy quark will have a component perpendicular to the velocity of the quark, even as seen in the local fluid rest frame. This perpendicular force can be large; we found instances where at early times, before hydrodynamization, its peak value in the local fluid rest frame was about half as large as the maximum drag force acting parallel to the velocity of the quark. The perpendicular force is nonzero at late times too, when the quark is propagating through a liquid that is described well by viscous hydrodynamics. This perpendicular force can also be attributed to the presence of gradients in the fluid velocity. Here too, it would be interesting to use a model of heavy quark dynamics in heavy ion collisions to investigate the consequences of these effects. That being for the future, at present the fourth lesson from our results is that the straightforward approach to modelling heavy quark dynamics in heavy ion collisions should be used with caution for heavy quarks at high rapidity.

From a more theoretical perspective, next steps that our results motivate include repeating our analysis for the collisions of sheets of energy with varying widths and seeking an analytical understanding of the effects of gradients in the fluid velocity (and temperature) on heavy quark energy loss.

\section{Acknowledgments}

We would like to thank Jorge Casalderrey-Solana, Michal Heller, Hong Liu, David Mateos, Stanislas Mrowczynski, Juan Pedraza, Silviu Pufu, Wilke van der Schee and Urs Wiedemann for helpful discussions. This work was supported by the U.S. Department of Energy under cooperative research agreement DE-FG0205ER41360. 


\section{A Trailing string solution in an equilibrium background}

The trailing string solution that describes an infinitely heavy quark being dragged in the $\vec{x}$-direction with velocity $\vec{\beta}$ through an equilibrium plasma with temperature $T_{\text {background }}$ is $[1,2]$

$$
\vec{x}=\vec{\beta}\left(t+u_{h} \tan ^{-1} \frac{u}{u_{h}}\right),
$$

where we have not yet made the transformation (2.5) meaning that the event horizon of the black brane in the bulk is located at $u_{h}=1 /\left(\pi T_{\text {background }}\right)$. With this choice of string profile, when we choose worldsheet coordinates of the form (2.10) the temporal constraint (2.13a) becomes a differential equation for $t$ as a function of $\tau$ and $u$ that, in the case of a time-independent metric corresponding to a static plasma with temperature $T_{\text {background }}$, has the solution

$$
t+u_{h} \tan ^{-1} \frac{u}{u_{h}}=\tau+u_{h} \sqrt{\gamma} \tan ^{-1} \frac{u \sqrt{\gamma}}{u_{h}}
$$

where $\gamma=1 / \sqrt{1-\vec{\beta}^{2}}$. After we make the transformation (2.5), the trailing string profile (A.1) is given by

$$
\vec{x}=\vec{\beta}\left(t+u_{h} \tan ^{-1} \frac{u}{(1+u \xi) u_{h}}\right)
$$

and for the case of a static plasma the solution to the temporal constraint equation is now

$$
t+u_{h} \tan ^{-1} \frac{u}{(1+u \xi) u_{h}}=\tau+u_{h} \sqrt{\gamma} \tan ^{-1} \frac{u \sqrt{\gamma}}{(1+u \xi) u_{h}} .
$$

As we have described in section 2.2, upon making the transformation (2.5) we choose worldsheet coordinates in which $u=\sigma$. This together with the expressions (A.3) and (A.4) give us our initial conditions for $X^{M}$, which is to say $u, t$ and $\vec{x}$, as functions of $\tau$ and $\sigma$.

If all we were interested in doing was dragging a heavy quark through static plasma, there would be nothing to add. The background of interest to us, however, is one where the heavy quark is initially in a region of spacetime filled with static plasma but in which in the future the quark will be slammed by two sheets of energy, incident upon it from the $+z$ and $-z$ directions. As we mentioned in section 2.2, at any given early Eddington-Finkelstein time $t$, even well before the sheets of energy collide on the boundary, the gravitational shocks are already colliding somewhere deep in the bulk. Eddington-Finkelstein coordinates on the worldsheet are advantageous from the point of view of making it possible to solve the evolution equations but from the point of view of specifying the initial conditions this feature is a complication. There are two possible ways to proceed. The route that we have followed in obtaining all the results that we show is to use (A.3) and (A.4) as our initial conditions at all $\sigma$, even deep in the bulk, even though deep in the bulk where the gravitational shocks are already colliding at the early $t$ at which we are initializing the string profile the choice (A.4) does not satisfy the temporal constraint equations. The other option is to use (A.3) and then to solve the temporal constraint differential equation numerically, replacing (A.4) by a numerically determined $t(\tau, \sigma)$. Because we are initializing at a time 
$t$ when the quark at the boundary is in a region of static plasma, these two options yield identical results close to the boundary, where (A.4) itself solves the temporal constraint equation. They are inequivalent deep in the bulk, and indeed there is no one "right answer" for how to initialize the string profile deep in the bulk in Eddington-Finkelstein coordinates. We have checked, however, that these two options yield identical results for the drag force on the heavy quark, meaning that for our purposes they are equivalent. The reason that the distinction between them is irrelevant is that it arises only deep enough within the bulk that well before the sheets collide at the boundary the region of the string that is affected by these considerations has been enveloped by the event horizon of the black brane. Because of this, the initial violation of the temporal constraint equation (assuming we pursue the first option) and the initial arbitrariness associated with choosing the profile (A.3) where there is no reason to do so (in either the first or second option) are causally disconnected from the boundary. Since the drag force on the heavy quark is computed from the near-boundary asymptotics of the string profile, nothing in this paragraph affects it.

\section{B Varying temperature}

We discovered in section 3.1 that when the energy density and pressures of the matter through which the heavy quark is moving change there seems to be a time delay in the response of the drag force experienced by the heavy quark, relative to the way in which the drag force would change if it were given by its value in a static plasma with the same instantaneous energy density or pressure. In this appendix we shall quantify this time delay in a time-dependent background that is much simpler than the colliding sheets of energy that are our focus throughout the rest of this paper. We shall consider a background that, at all times, is a spatially homogeneous plasma whose properties are exactly as if it is in thermal equilibrium at temperature $T$, and in particular which always has zero fluid velocity throughout, but by hand we shall make $T(t)$ change with time. This of course violates energy conservation in the boundary theory. It is therefore no surprise that the bulk metric that provides the dual gravitational description of this ad hoc setup is not a solution to Einstein's equations. The setup in this appendix is therefore not a model for anything; it is simply a device with which to evaluate the time delay in the response of the drag force on a heavy quark to a change in the conditions in which the heavy quark finds itself. Note that in this setup there are no spatial gradients of the fluid velocity since $u^{\mu}=(1,0,0,0)$ at all times. Also, $T_{e}=T_{\perp}=T_{\|}=T(t)$ at all times.

The metric that describes a spatially homogeneous plasma with a time-dependent temperature is given in Eddington-Finkelstein coordinates by (2.4) with

$$
\begin{aligned}
& A=\left(\left(\frac{1}{u}+\xi(t)\right)^{2} f(u, t)-2 \frac{\partial \xi}{\partial t}\right), \\
& \Sigma=\frac{1}{u}+\xi(t), \quad B=F=0,
\end{aligned}
$$

with

$$
f(u, v)=1-\left(\frac{u}{(1+u \xi(t)) u_{h}(t)}\right)^{4}
$$




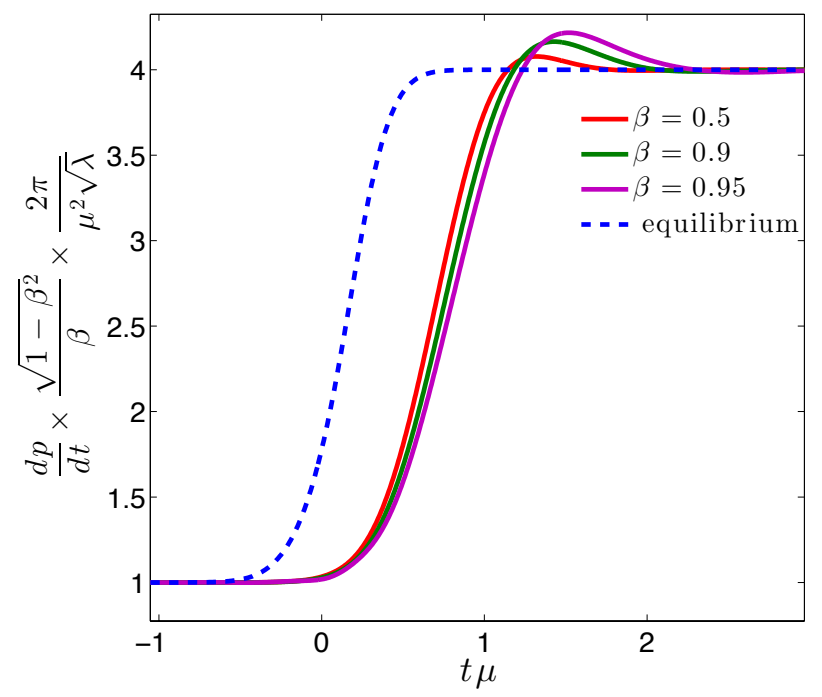

Figure 19. Drag force, scaled by $\beta \gamma$, for a heavy quark with velocity $\beta$ moving through a spatially homogeneous plasma with a time dependent temperature $T(t)$ that increases from $\pi T=\mu$ at early times to $\pi T=2 \mu$ at late times and is described by (B.4). The different solid curves show the scaled drag force on quarks with different velocities. The dashed curve is the equilibrium expectation (3.1). Because $T_{e}=T_{\perp}=T_{\|}=T(t)$ there is only a single dashed curve.

where $u_{h}(t)=1 /(\pi T(t))$ and where $\xi(t)$ describes the residual diffeomorphism introduced in (2.5). As we discussed there, we fix $\xi$ by demanding that the apparent horizon be at $u=1$. In this simplified setup, in coordinates in which $\xi=0$ the apparent horizon is at $u=u_{h}(t)$ whereas ensuring that it is at $u=1$ corresponds to choosing

$$
\xi(t)=\frac{1}{u_{h}(t)}-1
$$

which ensures that $f(u=1, t)=0$.

We shall choose a time-dependent temperature that starts at $\pi T=\mu$ at early times and ends at $\pi T=2 \mu$ at late times, rising smoothly during a window in time that is $\sim 1 /(k \mu)$ wide, with $k$ a parameter. We choose

$$
u_{h}(t)=1-\frac{1}{4}(1+\operatorname{erf}(k t \mu)) .
$$

For large values of $k, u_{h}(t)$ (and therefore $T(t)$ ) becomes a step function at $t=0$. In figure 19, we choose $k=3$. We have plotted the drag force rescaled in such a way that the dashed curve, which is obtained by substituting $T(t)$ into (3.1) and shows what the drag force would be in an equilibrium plasma with the instantaneous temperature $T(t)$, is independent of the quark velocity $\beta$ and is a plot of $(\pi T(t) / \mu)^{2}$. We see that the actual drag force, shown as the solid curves, is $\beta$-dependent even when rescaled as in the figure and shows a significant, and somewhat $\beta$-dependent, time delay. The temperature of the plasma has risen quite significantly before the drag force begins to rise; once the 


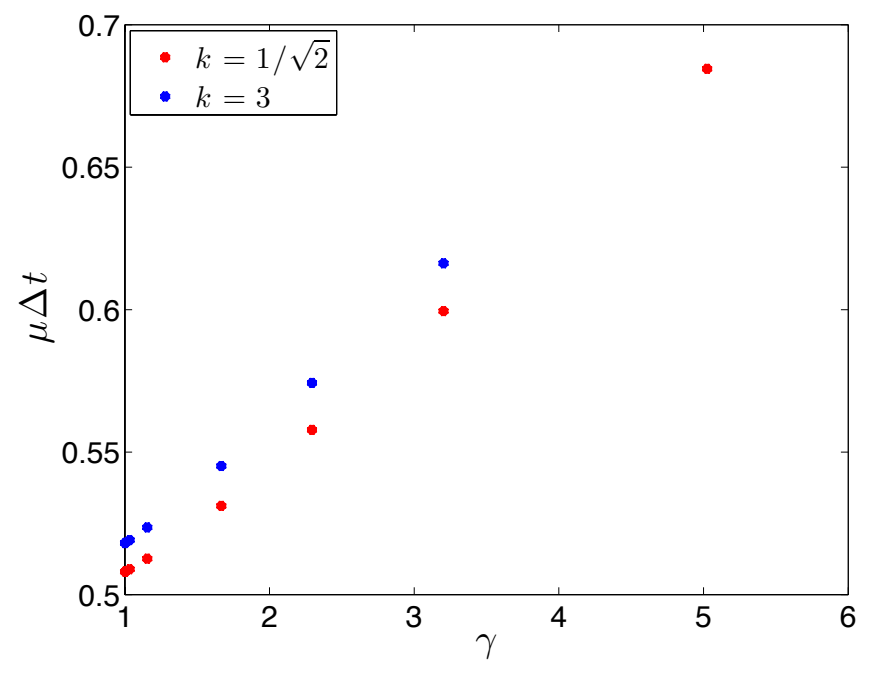

Figure 20. The delay time $\Delta t$, defined from results as in figure 19 as described in the text, as a function of $\gamma$ for two values of the parameter $k$ that controls the rapidity with which the temperature increases.

temperature reaches its final plateau the drag force is only about half way up its rise; and, finally, the drag force over-shoots before approaching its new equilibrium value from above.

There are various ways in which we could choose an operational definition of the time delay from the results in figure 19. We shall define the time delay $\Delta t$ as the delay between the times when the dashed and solid curves cross the midpoint between the initial drag force and the final drag force, i.e. in figure 19 when they cross 2.5. In figure 20 we show how $\Delta t$ depends on the quark velocity for two different values of $k$, the parameter that controls the rapidity with which $T(t)$ changes. For both values of $k$ we see that at low velocities there is a time delay of around 0.5 , which we note is around $1 /\left(\pi T_{\text {final }}\right)$ where $T_{\text {final }}$ is the final temperature. And, for both values of $k$ we see that the time delay increases with increasing $\beta$ in a way that is close to, but not exactly, linear with $\gamma$.

From a gravitational perspective, the fact that there is a time delay can be described in qualitative terms as if when the horizon at $u=u_{h}$ moves the drag force only learns of this at a time that is delayed by of order the light-travel-time for information from the horizon travelling through the bulk to reach the near-boundary region, where the drag on the heavy quark is encoded. If taken literally, this interpretation would suggest that in the setup of this appendix the time delay should start out around $1 /\left(\pi T_{\text {initial }}\right)$ and then drop to around $1 /\left(\pi T_{\text {final }}\right)$. This interpretation should not be taken too literally, however, because making $u_{h}$ time dependent makes the entire bulk metric at all $u$ time dependent, not just the metric near the horizon.

Open Access. This article is distributed under the terms of the Creative Commons Attribution License which permits any use, distribution and reproduction in any medium, provided the original author(s) and source are credited. 


\section{References}

[1] C. Herzog, A. Karch, P. Kovtun, C. Kozcaz and L. Yaffe, Energy loss of a heavy quark moving through $N=4$ supersymmetric Yang-Mills plasma, JHEP 07 (2006) 013 [hep-th/0605158] [INSPIRE].

[2] S.S. Gubser, Drag force in AdS/CFT, Phys. Rev. D 74 (2006) 126005 [hep-th/0605182] [INSPIRE].

[3] J. Casalderrey-Solana and D. Teaney, Heavy quark diffusion in strongly coupled $N=4$ Yang-Mills, Phys. Rev. D 74 (2006) 085012 [hep-ph/0605199] [InSPIRE].

[4] J.M. Maldacena, The Large-N limit of superconformal field theories and supergravity, Adv. Theor. Math. Phys. 2 (1998) 231 [Int. J. Theor. Phys. 38 (1999) 1113] [hep-th/9711200] [INSPIRE].

[5] E. Witten, Anti-de Sitter space and holography, Adv. Theor. Math. Phys. 2 (1998) 253 [hep-th/9802150] [INSPIRE].

[6] A. Karch and E. Katz, Adding flavor to AdS/CFT, JHEP 06 (2002) 043 [hep-th/0205236] [INSPIRE].

[7] J. Casalderrey-Solana, H. Liu, D. Mateos, K. Rajagopal and U.A. Wiedemann, Gauge/String Duality, Hot QCD and Heavy Ion Collisions, arXiv:1101.0618 [INSPIRE].

[8] P.M. Chesler and L.G. Yaffe, Holography and colliding gravitational shock waves in asymptotically AdS spacetime, Phys. Rev. Lett. 106 (2011) 021601 [arXiv:1011.3562] [INSPIRE].

[9] J. Casalderrey-Solana, M.P. Heller, D. Mateos and W. van der Schee, From full stopping to transparency in a holographic model of heavy ion collisions, arXiv:1305.4919 [INSPIRE].

[10] P.M. Chesler and L.G. Yaffe, Numerical solution of gravitational dynamics in asymptotically anti-de Sitter spacetimes, arXiv:1309.1439 [INSPIRE].

[11] R.A. Janik and R.B. Peschanski, Asymptotic perfect fluid dynamics as a consequence of AdS/CFT, Phys. Rev. D 73 (2006) 045013 [hep-th/0512162] [INSPIRE].

[12] P.M. Chesler and L.G. Yaffe, Horizon formation and far-from-equilibrium isotropization in supersymmetric Yang-Mills plasma, Phys. Rev. Lett. 102 (2009) 211601 [arXiv:0812.2053] [INSPIRE].

[13] P.M. Chesler and L.G. Yaffe, Boost invariant flow, black hole formation and far-from-equilibrium dynamics in $N=4$ supersymmetric Yang-Mills theory, Phys. Rev. D 82 (2010) 026006 [arXiv:0906.4426] [InSPIRE].

[14] I. Booth, M.P. Heller and M. Spalinski, Black brane entropy and hydrodynamics: The Boost-invariant case, Phys. Rev. D 80 (2009) 126013 [arXiv:0910.0748] [INSPIRE].

[15] M.P. Heller, R.A. Janik and P. Witaszczyk, The characteristics of thermalization of boost-invariant plasma from holography, Phys. Rev. Lett. 108 (2012) 201602 [arXiv:1103.3452] [INSPIRE].

[16] M.P. Heller, D. Mateos, W. van der Schee and D. Trancanelli, Strong Coupling Isotropization of Non-Abelian Plasmas Simplified, Phys. Rev. Lett. 108 (2012) 191601 [arXiv:1202.0981] [INSPIRE].

[17] W. van der Schee, Holographic thermalization with radial flow, Phys. Rev. D 87 (2013) 061901 [arXiv: 1211.2218] [INSPIRE]. 
[18] M.P. Heller, D. Mateos, W. van der Schee and M. Triana, Holographic isotropization linearized, JHEP 09 (2013) 026 [arXiv: 1304.5172] [INSPIRE].

[19] C. Shen, U. Heinz, P. Huovinen and H. Song, Systematic parameter study of hadron spectra and elliptic flow from viscous hydrodynamic simulations of $A u+A u$ collisions at $\sqrt{s_{N N}}=200$ GeV, Phys. Rev. C 82 (2010) 054904 [arXiv: 1010.1856] [inSPIRE].

[20] P.F. Kolb and U.W. Heinz, Hydrodynamic description of ultrarelativistic heavy ion collisions, in Quark gluon plasma, R.C. Hwa et al. eds. (2003), pg. 634 [nucl-th/0305084] [INSPIRE].

[21] U.W. Heinz, Thermalization at RHIC, AIP Conf. Proc. 739 (2005) 163 [nucl-th/0407067] [INSPIRE].

[22] M.E. Carrington, K. Deja and S. Mrowczynski, Parton Energy Loss in the Extremly Prolate quark-gluon Plasma, PoS(Confinement X) 175 [arXiv: 1301.4563] [INSPIRE].

[23] D. Grumiller and P. Romatschke, On the collision of two shock waves in AdS $S_{5}$, JHEP 08 (2008) 027 [arXiv: 0803.3226] [INSPIRE].

[24] S. de Haro, S.N. Solodukhin and K. Skenderis, Holographic reconstruction of space-time and renormalization in the AdS/CFT correspondence, Commun. Math. Phys. 217 (2001) 595 [hep-th/0002230] [INSPIRE].

[25] K. Skenderis, Asymptotically Anti-de Sitter space-times and their stress energy tensor, Int. J. Mod. Phys. A 16 (2001) 740 [hep-th/0010138] [INSPIRE].

[26] C.P. Herzog, Energy Loss of Heavy Quarks from Asymptotically AdS Geometries, JHEP 09 (2006) 032 [hep-th/0605191] [INSPIRE].

[27] P. Chesler, M. Lekaveckas and K. Rajagopal, Far-from-equilibrium heavy quark energy loss at strong coupling, Nucl. Phys. A904 (2013) 861c [arXiv:1211.2186] [INSPIRE].

[28] G. Giecold, Heavy quark in an expanding plasma in AdS/CFT, JHEP 06 (2009) 002 [arXiv:0904.1874] [INSPIRE].

[29] A. Stoffers and I. Zahed, Holographic Jets in an Expanding Plasma, Phys. Rev. C 86 (2012) 054905 [arXiv: 1110 . 2943] [INSPIRE].

[30] N. Abbasi and A. Davody, Moving Quark in a Viscous Fluid, JHEP 06 (2012) 065 [arXiv: 1202.2737] [INSPIRE].

[31] J. Bjorken, Highly Relativistic Nucleus-Nucleus Collisions: The Central Rapidity Region, Phys. Rev. D 27 (1983) 140 [inSPIRE].

[32] S. Peigne, P.-B. Gossiaux and T. Gousset, Retardation effect for collisional energy loss of hard partons produced in a QGP, JHEP 04 (2006) 011 [hep-ph/0509185] [INSPIRE].

[33] A. Guijosa and J.F. Pedraza, Early-Time Energy Loss in a Strongly-Coupled SYM Plasma, JHEP 05 (2011) 108 [arXiv: 1102.4893] [INSPIRE].

[34] M. Chernicoff, D. Fernandez, D. Mateos and D. Trancanelli, Drag force in a strongly coupled anisotropic plasma, JHEP 08 (2012) 100 [arXiv:1202.3696] [INSPIRE].

[35] A. Nata Atmaja and K. Schalm, Anisotropic Drag Force from 4D Kerr-AdS Black Holes, JHEP 04 (2011) 070 [arXiv: 1012.3800] [InSPIRE].

[36] K.B. Fadafan and H. Soltanpanahi, Energy loss in a strongly coupled anisotropic plasma, JHEP 10 (2012) 085 [arXiv: 1206.2271] [INSPIRE]. 
[37] G.D. Moore and D. Teaney, How much do heavy quarks thermalize in a heavy ion collision?, Phys. Rev. C 71 (2005) 064904 [hep-ph/0412346] [INSPIRE].

[38] H. van Hees, V. Greco and R. Rapp, Heavy-quark probes of the quark-gluon plasma at RHIC, Phys. Rev. C 73 (2006) 034913 [nucl-th/0508055] [INSPIRE].

[39] H. van Hees, M. Mannarelli, V. Greco and R. Rapp, Nonperturbative heavy-quark diffusion in the quark-gluon plasma, Phys. Rev. Lett. 100 (2008) 192301 [arXiv:0709.2884] [InSPIRE].

[40] Y. Akamatsu, T. Hatsuda and T. Hirano, Heavy Quark Diffusion with Relativistic Langevin Dynamics in the quark-gluon Fluid, Phys. Rev. C 79 (2009) 054907 [arXiv:0809.1499] [INSPIRE].

[41] R. Rapp and H. van Hees, Heavy Quarks in the quark-gluon Plasma, in Quark Gluon Plasma 4, R.C. Hwa, X.-N. Wang eds., World Scientific (2010), pg. 111 [arXiv:0903.1096] [INSPIRE].

[42] W. Alberico, A. Beraudo, A. De Pace, A. Molinari, M. Monteno et al., Heavy-flavour spectra in high energy nucleus-nucleus collisions, Eur. Phys. J. C 71 (2011) 1666 [arXiv:1101.6008] [INSPIRE]. 